Published in final edited form as:

Nat Rev Cancer. ; 11(8): 541-557. doi:10.1038/nrc3087.

\title{
Advances in sarcoma genomics and new therapeutic targets
}

\author{
Barry S. Taylor ${ }^{1,2}$, Jordi Barretina ${ }^{3,4}$, Robert G. Maki ${ }^{5}$, Cristina R. Antonescu ${ }^{1}$, Samuel \\ Singer ${ }^{1}$, and Marc Ladanyi $\mathbf{i}^{1,}$, \\ ${ }^{1}$ Memorial Sloan-Kettering Cancer Center, New York, New York 10065, USA \\ 2University of California, San Francisco, San Francisco, California 94143, USA \\ ${ }^{3}$ Dana-Farber Cancer Institute, Boston, Massachusetts 02115, USA \\ ${ }^{4}$ Broad Institute, Cambridge, Massachusetts 02142, USA \\ ${ }^{5}$ Mount Sinai School of Medicine, New York, New York 10029, USA
}

\section{Preface}

Increasingly, human mesenchymal malignancies are classified by the abnormalities that drive their pathogenesis. While many of these aberrations are highly prevalent within particular sarcoma subtypes, few are currently targeted therapeutically. Indeed, most subtypes of sarcoma are still treated with traditional therapeutic modalities and in many cases are resistant to adjuvant therapies. In this Review, we discuss the core molecular determinants of sarcomagenesis and emphasize the emerging genomic and functional genetic approaches that, coupled to novel therapeutic strategies, have the potential to transform the care of patients with sarcoma.

Sarcomas are uncommon yet diverse mesenchymal malignancies, arising in or from bone, cartilage, or connective tissues such as muscle, fat, peripheral nerves, fibrous, or related tissues (FIG. 1). Together, they affect $~ 11,000$ individuals in the United States each year and approximately 200,000 worldwide, arise from multiple lineages, and range from indolent to highly invasive and metastatic ${ }^{1,2}$. From a molecular genetics perspective, they have traditionally been classified into two broad categories, each of which includes clinically diverse sarcomas. First are those sarcomas with near-diploid karyotypes and simple genetic alterations including translocations or specific activating mutations. The second are tumors with complex and unbalanced karyotypes. These tumors are typified by genome instability resulting in multiple genomic aberrations in a single tumor's genome, and heterogeneity of aberrations across tumors of a given type. The contrasting features of these two categories, which we first highlighted in $2002^{3}$, have been well reviewed ${ }^{4}$. These categories are, however, broadly drawn and do not reflect the genetic diversity among tumors of a given type, the subtypes within classes, or their diverse tumor biology (FIG. 1).

\footnotetext{
${ }^{*}$ Correspondence to M.L.: ladanyim@ @ mskcc.org.

Competing interests statement

The authors declare no competing financial interests.

Further information

Sarcoma data portals: http://cbio.mskcc.org/cancergenomics/sarcoma/, http://www.broadinstitute.org/sarcoma/

The Cancer Genome Atlas (TCGA), studied cancers: http://cancergenome.nih.gov/wwd/cancers_studied_by_tcga.asp

Online Mendelian Inheritance in Man: http://www.ncbi.nlm.nih.gov/omim

Mitelman Database of Chromosome Aberrations and Gene Fusions in Cancer: http://cgap.nci.nih.gov/Chromosomes/Mitelman

NCI CTD 2 : http://ocg.cancer.gov/programs/ctdd.asp

The RNAi Consortium: http://www.broadinstitute.org/rnai/trc/

Human ORFeome: http://horfdb.dfci.harvard.edu/

National Cancer Institute Drug Dictionary: http://www.cancer.gov/drugdictionary/
} 
Most sarcomas with simple genetic alterations are translocation-associated sarcomas (approximately one-third of all sarcomas). These tumors tend to arise de novo and, in some cases, harbor only the single defining cytogenetic abnormality that is present at initiation and retained throughout their clonal evolution. The majority of gene fusions resulting from these specific translocations encode chimeric transcription factors that cause transcriptional dysregulation of target genes, while others encode chimeric protein tyrosine kinases or autocrine growth factors ${ }^{5}$. Although well studied, the physiological roles of the individual genes in these fusions have seldom been directly linked to their respective sarcoma phenotypes, save perhaps for translocations of the myogenic transcription factor genes paired box 3 (PAX3) and $P A X 7$ with forkhead box $\mathrm{O} 1$ (FOXO1) in alveolar rhabdomyosarcomas (ARMS; discussed below) ${ }^{6}$.

In contrast to translocation-associated sarcomas, some karyotypically complex sarcomas can arise from a less aggressive form and pass through discrete stages of progression accompanied by increasing genomic complexity. Examples include the progression from atypical lipoma or well-differentiated liposarcoma to dedifferentiated liposarcoma ${ }^{7-9}$, or from neurofibroma to malignant peripheral nerve sheath tumor (MPNST) ${ }^{10}, 11$, or from enchondroma to chondrosarcoma ${ }^{12}$. Importantly, however, most high-grade karyotypically complex sarcomas present de novo, without antecedent lower-grade lesions. A detailed listing of the genetic abnormalities in sarcomas and their conventional treatment is available elsewhere ${ }^{4,13}, 14$. We focus here on the core mechanisms of pathogenesis in soft tissue sarcoma, the advanced genomic and functional genetic approaches being deployed for target discovery in this group of diseases, and the novel therapeutic approaches for their treatment.

\section{Molecular mechanisms of sarcomagenesis}

The mechanisms that drive human sarcomagenesis fall into three broad categories: transcriptional dysregulation owing to aberrant fusion proteins resulting from genomic rearrangements (FIG. 2a), somatic mutations in key genes and signaling pathways, and DNA copy number abnormalities. The epigenetic mediators of sarcomagenesis are largely still to be determined, as while specific chromatin changes are implied by translocations and subsequent transcriptional dysregulation, data on recurrent methylation in sarcoma genomes is limited. Although this review focuses on these three core oncogenic mechanisms and the distinct therapeutic modalities that may follow, some consideration of pathogenetic mechanisms relating to chromosomal translocations and genomic complexity or instability in sarcomas may be in order. The perennial question of how and why translocations arise has been the subject of recent reviews ${ }^{15}$. In sarcomas, as in many leukemias, these appear to be fundamentally random events that become fixed through natural selection within the precursor cell. In silico analysis of sequence and structure indicated that features such as overall gene size, average intron length, and the length of the longest intron were all higher in translocation partner genes ${ }^{16}$. Additional factors increase the likelihood of random breaks in two genes leading to an illegitimate recombination event. These include increased availability of translocation partner genes created by open chromatin conformation associated with gene transcription or replication, or the unexpected proximity of some partner genes due to either the three dimensional arrangement of chromosomes in the nucleus ${ }^{17}$, or coordinated transcription within the same transcriptional hubs. Importantly, socalled recombinogenic DNA sequence elements may be anecdotally involved ${ }^{18}$, but are not more frequent in translocated genes. More recently, binding of a transcription factor, the androgen receptor $(A R)$, has been implicated more directly in generating DNA strand breaks and consequent gene fusions ${ }^{19-21}$, an observation so far restricted to prostate cancer, but with intriguing implications for other hormone-driven cancers. Finally, regarding external risk factors, sarcoma translocations, in particular the $t(X ; 18)$ of synovial sarcoma, may be rarely related to radiotherapy-induced DNA damage $22-24$. 
Another aspect of genomic integrity is the mechanisms of telomere maintenance of which two main types have been described in human tumors: telomerase activation and the alternative lengthening of telomeres (ALT). These appear to differ in frequency between the major genomic classes of sarcomas. A predominance of telomerase activation in the absence of ALT appears to characterize sarcomas with specific chromosomal translocations.

Alternatively, ALT is frequently seen in sarcomas with non-specific complex karyotypes ${ }^{25,26}$, and a connection between ALT and mesenchymal stem cell biology has been proposed ${ }^{27}$.

Sarcomas with non-specific complex karyotypes, but not translocation-associated sarcomas, are also occasionally seen in some hereditary syndromes associated with genomic instability such as Werner syndrome (gene: $W R N)^{28}$, Nijmegen breakage syndrome (gene: $\left.N B S 1\right)^{29}$, and Rothmund-Thomson syndrome (gene: RECQL4) ${ }^{30,31}$. Finally, recent low-coverage whole genome sequencing found that 3/9 osteosarcomas and 2/11 chordomas underwent a process termed chromothripsis. Rather than a multistep accumulation of unbalanced rearrangements, this is a single catastrophic genomic instability event affecting primarily a single chromosome $\mathrm{e}^{32}$. Investigating the pathogenesis of chromothripsis and its occurrence in other sarcomas is of immediate interest.

\section{Transcriptional target dysregulation}

Most translocation-associated sarcomas share a common biology of transcriptional target dysregulation. As noted above, most recurrent tumor-type-specific translocations in sarcomas produce gene fusions that encode aberrant transcriptional proteins. The general biology of cancer gene fusions has been well reviewed ${ }^{33}$. Likewise, general reviews of translocation-associated sarcomas, including comprehensive listings of recurrent gene fusions in sarcomas, have recently been published (FIG. 2a) ${ }^{5,14}$. Here, we will limit ourselves to two aspects of transcriptional target gene dysregulation in translocation sarcomas that have been the focus of recent advances: the application of genome-wide transcription factor location analyses to comprehensively identify target genes of the fusion proteins, and the emerging evidence for aberrant nuclear reprogramming of mesenchymal stem cells in translocation-associated sarcomas. In the Therapeutic Avenues section below, we also discuss the use of the transcriptional targets of the fusion proteins as therapeutic targets, a third area of recent advances.

Genome-wide approaches to define the target gene repertoires of sarcoma fusions have included chromatin immunoprecipitation (ChIP) coupled to arrays (ChIP-on-chip), and, more recently, to second-generation sequencing (ChIP-seq: Box 1). Both methods identify binding sites for the aberrant fusion proteins, but ChIP-seq, unlike ChIP-on-chip using commercial promoter arrays, is not limited to regions surrounding promoters. Upon integration with expression profiles, one can determine whether the effect of a given fusion is predominantly repressive or activating.

\section{Box 1}

\section{Advances in cancer genome and transcriptome characterization}

Second-generation sequencing is enabling nucleotide-resolution oncogenomics ${ }^{202}$. Paired-end (or mate-paired) sequencing involves sequencing of short stretches of DNA on both ends of a larger fragment and aligning to the reference genome. Atypically aligned pairs (those with unexpected position, orientation or separation distance) often reflect genomic rearrangements such as translocations (FIG. 2b). Paired-end sequencing is therefore a powerful method for ascertaining structural rearrangements and marks the first time this information is readily available in an unbiased manner. It is suitable for the 
detection of rearrangements from variable depth-of-coverage whole-genome sequencing, and its sensitivity increases as the fragment length increases. These methods will help detect previously unknown 'driver' fusions in sarcomas with highly complex karyotypes. Paired-end sequencing can also be deployed in RNA sequencing of tumor transcriptomes, as has been done for prostate cancers and other malignancies ${ }^{203,204}$. To explore the biology of chimeric transcriptional proteins in translocation-associated sarcomas, chromatin immunoprecipitation coupled to sequencing (ChIP-seq) can determine fusion protein location, facilitating target gene discovery (see main text). Finally, deep wholeexome and whole-genome sequencing, while described in detail elsewhere ${ }^{202}$, have a central place in sarcoma genomics. Exome sequencing first captures and then deeply sequences all protein-coding exons of human genes. By contrast, whole-genome sequencing is unbiased, sequencing all accessible nucleotides in the human genome. Both experiments detect point mutations and small insertions and deletions (indels) in exons, while whole-genome sequencing can simultaneously capture genome structure (in pairedend format, described above) and critically, intergenic variation. Intergenic germline variation or somatic mutations, while under-explored currently, could play an important role in sarcomagenesis. This is typified by the $M D M 2^{S N P 309}$ promoter polymorphism ${ }^{205}$, which along with $M D M 2$ amplification and TP53 deletion and mutation, represents another mechanism of aberrant p53 activity in a broad range of sarcomas.

Mapping the genomic binding sites of the PAX3-FOXO1 fusion protein in ARMS cells has shown that binding is associated with activation of transcription ${ }^{34}$. PAX3-FOXO1 binds primarily to PAX3 sites outside of the immediate vicinity of transcription start sites, typically $>4$ kilobases $(\mathrm{kb})$ downstream. Co-enrichment of target PAX3 motifs with E-box motifs suggests co-regulation of many target genes by other transcription factors that bind Eboxes $^{34}$. The direct targets identified include myogenic genes such as myogenic differentiation 1 (MYODI) and myogenic factor 5 (MYF5), as well as many biologically interesting targets such as fibroblast growth factor receptor 4 (FGFR4), anaplastic lymphoma kinase $(A L K), M E T$, insulin-like growth factor 1 receptor $(I G F 1 R)$, and $M Y C N$, in some cases confirming previous single-gene studies ${ }^{35-37}$. The role of some of these PAX3-FOXO1 target genes in sarcomagenesis is further discussed below.

In alveolar soft part sarcoma (ASPS), the ASPL (also known as ASPSCRI) gene fuses with the transcription factor binding to IGHM enhancer 3 (TFE3) gene to form a chimeric protein that retains the TFE3 DNA binding domain and therefore its CACGTG recognition site. In ChIP-on-chip studies, we have found ASPL-TFE3 localization is predictably enriched at this canonical site and exclusively associated with target gene activation, including $M E T^{38}$, cytochrome P450 17A1 (CYP17A1) and uridine phosphorylase 1 (UPPI) ${ }^{39}$.

A somewhat more complicated picture has emerged for the major Ewing sarcoma fusion involving EWSR1 (also known as EWS) and the Friend leukaemia virus integration 1 (FLII) gene. Several ChIP datasets have been generated in different Ewing sarcoma cell lines with endogenous EWS-FLI1, all using the same FLI1 antibody for immunoprecipitation of EWSFLI1-bound DNA. The numbers of bound genomic regions in such studies have varied widely ${ }^{40-42}$. ChIP-seq subsequently demonstrated that the majority of genomic regions bound by EWS-FLI1 were intergenic and that, through its FLI1-derived ETS family DNAbinding domain, EWS-FLI1 binds avidly to GGAA microsatellites ${ }^{40,41}$. Microsatellites containing 6 or more GGAA repeats (the core ETS domain binding sequence) are associated with EWS-FLI1 target gene upregulation ${ }^{40,42}$. These repeats are often more than $200 \mathrm{~kb}$ upstream of the target gene transcription start site, suggesting that chromatin looping brings distant regions together in a transcriptional hub to allow EWS-FLI1 to modulate gene expression. As microsatellites are known polymorphic sites, it has been hypothesized that 
higher repeat content at one or more key target genes may underlie individual or ethnic differences in Ewing sarcoma susceptibility, for instance its rarity in individuals of African descent $^{41}$.

EWS-FLI1 also binds to more conventional, non-repetitive ETS motifs, and these sites are associated with genes that show either repression or activation of transcription ${ }^{42}$. A subset of EWS-FLI1 target regions show co-enrichment of sites for E2F, nuclear respiratory factor 1 (NRF1), and nuclear transcription factor Y (NFY) raising the possibility of specific cooperative interactions ${ }^{43}$. In general, the combination of genome-wide target gene identification with gene expression data should accelerate the discovery of genes crucial to tumor growth and survival in translocation sarcomas. Genes found to be directly upregulated by specific aberrant sarcoma fusion proteins can be subjected to focused RNA interference (RNAi)-based screens to identify the genes most essential to the sarcoma in question (see Target Discovery below).

\section{Reprogramming}

Recent efforts to generate non-embryonic stem cells have renewed interest in nuclear or lineage reprogramming 44,45 . Understanding reprogramming may also inform our concepts of translocation sarcomas driven by aberrant transcription factors. Assigning lineage to translocation sarcomas has proven difficult, as is the case for Ewing and synovial sarcoma, ASPS, and others. The cell-of-origin for each of these has long been debated, especially owing to another peculiar clinical feature of these sarcoma types: their occurrence in unusual sites for tumors of bone and soft tissue, such as kidney, lung, or pancreas. One explanation for both characteristics is an origin from more than one stem or progenitor cell type or from related precursor cells in different parts of the body, with the similar undifferentiated or aberrantly differentiated phenotypes resulting from nuclear reprogramming by the aberrant transcription factors. For example, it has been shown that EWS-FLI1, the fusion defining Ewing sarcoma, can induce neuroectodermal gene expression in heterologous cell types such as fibroblasts and rhabdomyosarcoma cells ${ }^{46,47}$.

Indeed, this scenario was postulated previously ${ }^{48}$, and is supported by compelling data from recent studies, although some disagreements remain ${ }^{49}$. Silencing EWS-FLI1 in Ewing sarcoma cell lines produces an expression profile most similar to mesenchymal stem cells (MSCs) or mesenchymal progenitor cells ${ }^{50,51}$ and these can subsequently be induced to differentiate along adipogenic or osteoblastic lineages ${ }^{51}$. Thus, EWS-FLI1 induces a limited neuroectodermal gene expression program and imposes a differentiation block on MSCs (or a related cell type ${ }^{52}$, including a block on osteogenic differentiation by inhibiting runtrelated transcription factor 2 (RUNX2) binding to genes associated with osteogenic differentiation ${ }^{53}$. In the converse experiment, EWS-FLI1 expression in human MSCs induces a Ewing sarcoma gene expression profile, especially clear in MSCs derived from younger individuals ${ }^{54,55}$. By contrast, EWS-FLI1 expression in differentiated cell types with an intact ARF-p53 pathway induces apoptosis or growth arrest ${ }^{46}$. In human MSCs, EWS-FLI1 directly upregulates the polycomb group repressor enhancer of zeste homolog 2 $(E Z H 2)^{56}$ and induces expression of embryonic stem cell genes POU5F1 (also known as OCT4), SRY-box 2 (SOX2) and NANOG, at least partly by repressing $m i R-145$ expression $^{54}$. Interestingly, EWSR 1 also fuses with POUSF1 itself, albeit rarely, in undifferentiated bone sarcoma ${ }^{57,58}$, myoepithelial tumors of the soft tissue ${ }^{59}$, and in certain salivary gland tumors ${ }^{60}$.

Synovial sarcomas contain fusions of the $S S 18$ (also known as $S Y T$ ) gene with either $S S X 1$ or $S S X 2$. In a striking analogy to the EWS-FLI1 data, synovial sarcoma cell lines also express POU5F1, SOX2 and NANOG, and silencing of SYT-SSX in these cell lines enhances their potential to differentiate along adipogenic, osteoblastic or chondrogenic 
lineages ${ }^{61}$. The formation of synovial sarcoma-like tumors in mice with conditional expression of SYT-SSX2 in myoblasts ${ }^{62}$ or other lineages ${ }^{63}$ can be interpreted as further evidence of nuclear reprogramming by the fusion protein in a variety of more or less committed mesenchymal lineages. Finally, the sarcoma fusions of myxoid liposarcoma [fused in sarcoma (FUS)-DDIT3 (also known as CHOP)] and ARMS (PAX3-FOXO1) have also been reported to transform mouse mesenchymal stem or progenitor cells ${ }^{64,65}$.

\section{Mutations in key genes and signaling pathways}

Excluding the gene fusions in translocation sarcomas, few highly recurrent driver genes have been described in sarcoma. The major exception here is gastrointestinal stromal tumor (GIST). GIST, one of the more common human sarcoma types, is characterized by oncogenic mutations in $K I T$, or less often in platelet-derived growth factor receptor-a (PDGFRA), or rarely in $B R A F^{66-68}$. In fact, the dependence of GIST on constitutively activated KIT and PDGFRA has led to treatment with selective kinase inhibitors (discussed below), representing a paradigm of targeted therapy in solid tumors. Oncogenic mutations occur in several different domains of KIT, and the location affects sensitivity to targeted inhibitors. Levels of KIT are also high in interstitial cells of Cajal, the presumed cell of origin for GIST. Nevertheless, oncogenic KIT mutations (in the activation domain; D816V in particular) are also found in tumors of diverse lineages including mastocytosis, acute myeloid leukemia, and germ cell tumors.

Approximately $10 \%$ of adult GISTs lack a KIT or PDGFRA mutation, a small subset $(<1 \%$ of total GIST cases) harbor BRAF-V600E mutations (Table 1) ${ }^{66}$. Most pediatric GISTs harbor no mutations in KIT, PDGFRA, or BRAF, although KIT pathway activity is high in pediatric cases and in adult cases lacking mutations. In total, approximately $10 \%$ of adult and most pediatric GISTs harbor no mutations in KIT, PDGFRA, or BRAF, although KIT pathway activity is high. Among these, pediatric tumors show consistent overexpression of $I G F 1 R$ mRNA and protein, although the mechanism remains unknown as no genomic amplifications or activating mutations have been described at the IGFIR locus. In fact, pediatric tumors have mostly diploid genomes with few if any DNA copy-number alterations ${ }^{69}$. Therefore, ongoing deep sequencing in pediatric GISTs is expected to identify alternative oncogenic events. A particularly attractive method may be hybrid capture of protein-coding exons followed by second-generation sequencing (exome sequencing: see Box 1) given the power of its completeness and suitability for profiling small patient numbers.

Although generally sporadic, GIST can also present as part of syndromes such as familial GIST, Carney's triad, Carney-Stratakis syndrome, and neurofibromatosis. In CarneyStratakis syndrome, which is characterized by the co-occurrence of GIST and paraganglioma, germline mutations in genes encoding subunits of succinate dehydrogenase have been identified, as is also the case in familial paraganglioma ${ }^{70}$. Most GISTs occurring in association with neurofibromatosis type I harbor somatic inactivation of the wild-type neurofibromin 1 (NFI) allele, while very few have KIT or PDGFRA mutations ${ }^{71,72 .}$

A role for tyrosine kinases is also emerging in angiosarcoma, a highly aggressive vascular tumor, where transcriptional profiles show striking overexpression of vascular-specific receptor tyrosine kinases including kinase insert domain receptor $(K D R$; which encodes vascular endothelial growth factor receptor 2 (VEGFR2)), TIE1, SNF related kinase $(S N R K), T E K$, and fms-related tyrosine kinase $1(F L T 1)^{73}$. Sequencing of these five genes revealed $K D R$ mutations in about $10 \%$ of cases of angiosarcoma. The VEGFR2 mutant proteins, when expressed in COS-7 cells, showed ligand-independent activation ${ }^{73}$. 
A recent large-scale analysis of the genomic landscape of sarcomas encompassing seven major subtypes (myxoid/round-cell, dedifferentiated, and pleomorphic liposarcomas; myxofibrosarcoma, leiomyosarcoma, GIST, and synovial sarcoma) identified frequent mutations in TP53 (which encodes p53), NF1, and PI3K catalytic subunit-a $(P I K 3 C A)^{74}$. TP53 mutations were identified in $17 \%$ of pleomorphic liposarcomas, consistent with these mutations being frequent in sarcomas with complex karyotypes ${ }^{75,76}$. By contrast, in translocation-associated sarcomas secondary genetic alterations, such as TP53 mutations or homozygous deletions of cyclin-dependent kinase inhibitor $2 \mathrm{~A}(C D K N 2 A)$, are less common but, when present, are associated with a highly aggressive clinical course ${ }^{77}$. The discovery of PIK3CA mutations in 18\% of myxoid/round-cell liposarcomas (Table 1) raises the possibility that secondary mutations may cooperate with the FUS-CHOP fusion protein in oncogenesis ${ }^{74}$. PIK3CA mutations clustered in the same two hot spots observed in epithelial tumors: the helical domain (E542K and E545K) and the kinase domain (H1047L and H1047R). Patients with helical domain mutations had a shorter disease-specific survival and increased AKT phosphorylation at both CREB-regulated transcription coactivator 2 (TORC2; also known as CRTC2) and pyruvate dehydrogenase kinase 1 (PDK1) phosphorylation sites than those with wild-type or kinase-domain-mutant tumors ${ }^{74}$.

Another novel finding is that of $N F 1$ alterations (point mutations or deletions) in $10 \%$ of myxofibrosarcomas and $8 \%$ of pleomorphic liposarcomas ${ }^{74}$ (Table 1). $N F 1$ germline and somatic mutations are typically associated with NF1 inactivation in sarcomas in individuals with neurofibromatosis type 1 syndrome, but NF1 mutations had not been previously described in sporadic sarcomas.

\section{Genomic copy-number alterations}

DNA copy-number alterations are the third core mechanism of sarcomagenesis. Sarcomas span a wide range of complexity among human malignancies in their copy-number alterations ${ }^{78}$. They vary from translocation-associated sarcomas with generally few copynumber alterations, either broad or focal, to karyotypically complex subtypes that are heterogeneous, unstable and profoundly altered in genomic copy number. In addition, a recent high-resolution array-based copy-number analysis revealed a category with intermediate complexity mainly characterized by few, yet highly recurrent amplifications, exemplified by dedifferentiated liposarcomas ${ }^{74}$. These and similar genomic data support an alternative sarcoma classification to the one based on low-resolution karyotypes. These three groups are genomically simple sarcomas, driven by pathognomonic translocations or point mutations; non-translocation-associated sarcomas of intermediate genomic complexity; and highly genomically complex sarcomas, while some subtypes may not fit so neatly in these broad groups, such as PAX7-FOXO1-positive ARMS. Data from another copy-number analysis show that the third category can be subdivided into sarcomas with few chromosome arm or whole chromosome gains or losses and sarcoma genomes with a high level of chromosomal complexity ${ }^{79}$.

The first group, genomically simple sarcomas, harbor characteristic gene fusions or activating mutations thought to represent early events in their pathogenesis. Yet even these tumors can acquire genomic complexity in advanced stages of disease ${ }^{80,81}$.

Intermediate complexity sarcomas are exemplified by well-differentiated and dedifferentiated liposarcomas, which are driven mainly by chromosome 12 alterations, often generating extra-chromosomal episomes, ring chromosomes and larger markers ${ }^{82}$ (FIG. 2b). These 12q gains have high prevalence (80-90\%) and co-amplified oncogenes cyclindependent kinase $4(C D K 4)$ and $M D M 2$ can serve as confirmatory diagnostic markers ${ }^{83}$ and as potential pharmacological targets ${ }^{74,84,85}$. The structure, stability and reintegration of these amplicons into liposarcoma genomes can alter their affect on oncogenic phenotypes as 
well ${ }^{86}$. Another gene affected by $12 \mathrm{q}$ amplification is $H M G A 2$, which often loses its $3^{\prime}$ untranslated region (UTR), disrupting microRNA-mediated repression ${ }^{87}$. This genomic remodeling of chromosome 12 is likely the result of progressive rearrangement and amplification in an evolving amplicon rather than a single catastrophic event such as the recently proposed chromothripsis, seen in a subset of osteosarcomas and chordomas (Table $1)^{32}$. Similar 12q amplifications occur at lower frequencies in other mesenchymal tumors such as osteosarcomas ${ }^{88}$ as well as several epithelial tumor types ${ }^{78}$.

Other notable, albeit less recurrent amplifications, in intermediate-complexity sarcomas occur on $1 \mathrm{p}$ and $6 \mathrm{q}$. These amplifications, which appear to be mutually exclusive, span genes in the p38 and JNK pathways of MAPK signaling including, on 1p, JUN(Table 1) and, on 6q, TAB2 and MAP3K5 (also known as $A S K 1$ ), a kinase upstream of JUN ${ }^{9,89-91}$. An additional target of genomic amplification is telomerase reverse transcriptase (TERT) $(\text { on } 5 \mathrm{p})^{74}$. Some targets of genomic amplification appear to be shared among a subset of both intermediate and highly complex sarcomas, including Yes-associated protein 1 (YAPI) and vestigial like 3 (VGLL3) on 11q22 and 3p12, respectively ${ }^{92}$.

Finally, highly complex sarcomas harbor multiple numerical and structural chromosome aberrations that are reminiscent of the vast majority of epithelial tumors. Molecular classification of these subtypes reflect varying levels of similarity in their genomic aberrations; some subtypes may be considered a single entity ${ }^{93}$, while others are distinct ${ }^{94}$. Broad amplifications of several chromosome arms (such as $5 \mathrm{p}^{95}$ ) often occur in combination with deletions affecting well-established tumor suppressors such as $C D K N 2 A, C D K N 2 B$, $P T E N$, retinoblastoma 1 (RBI), NF1 and TP53. The affected gene, if not homozygously deleted, often harbors an inactivating mutation in the remaining allele ${ }^{74}$. In fact, several of these genes have a direct role in maintaining chromosome integrity 96,97 and their loss of function may be an early event leading to genomic instability in highly complex sarcomas. In other subtypes, such as leiomyosarcoma, genomic deletions are more common than amplifications ${ }^{74,98}$. Nevertheless, at least a subset of leiomyosarcomas depends on the specific amplification of myocardin $(M Y O C D)$, which encodes a smooth muscle-specific transcriptional coactivator of the serum response factor (SRF) (Table 1) ${ }^{99-101}$. The involvement of $M Y O C D$ in smooth muscle differentiation implies it may serve as a lineagesurvival oncogene ${ }^{102}$. Therefore, while systematic catalogues of copy numbers alterations point to pathways potentially activated in specific subtypes, to precisely delineate genes involved in these events that drive sarcomagenesis it will be essential to annotate genomic characterization with high-throughput functional genetics for target discovery.

\section{Target Discovery}

Systematic surveys of cancer genomes with integrated genomics have proven an effective approach in identifying targetable genetic alterations in specific cancer types. The list of potential targets is expected to grow with the expanded use of second-generation sequencing technologies, which detect not only genome-wide copy-number changes, but rearrangements and mutations (Box 1).

Thus far, large genomic characterization efforts in cancer have mainly focused on epithelial and haematological cancers. Given the need for new therapies for sarcomas, their inclusion in such studies is expected in the near future. For instance, the Cancer Genome Atlas (TCGA) project is initiating a comprehensive genomic analysis of dedifferentiated liposarcoma, leiomyosarcoma and undifferentiated pleomorphic sarcoma, although this effort will have to overcome a perennial challenge in sarcoma genomic research: the scarcity of samples. Nevertheless, given the large number of differentiation lineages among diverse 
sarcomas, a detailed genetic characterization of these tumors is likely to benefit our wider understanding of cancer in general.

\section{Genomics-guided functional genetics}

A gene recurrently altered in a sarcoma subtype does not necessarily play a role in cancer initiation or progression. In fact, the identification of recurrent lesions (Box 2) far outstrips our ability to test their importance. To determine the involvement of a gene in sarcoma biology and to credential it as a therapeutic target, systematic biological validation in genetically defined models must follow. Furthermore, even when a causal role for a given genetic alteration is experimentally supported in a particular cancer type, the critical downstream targets may remain elusive, requiring further functional studies. Yet, functional studies in sarcoma are hampered by the dearth of such appropriate models. Only limited numbers of human sarcoma cell lines exist, in part because of the rarity of certain diagnoses and resulting scarcity of samples. Moreover, for each of the subtypes with complex genomes, multiple cell lines are needed to represent the diversity of genetic alterations within that subtype. Several large-scale projects now aim to genetically characterize large numbers of human cancer cell lines and screen these against a range of anti-cancer therapies to correlate drug sensitivity with genetic markers. Among these are the Cancer Cell Line Encyclopedia (J.B. personal communication) and the Sanger Cancer Cell Line Project ${ }^{103}$, the latter is assembling approximately 800 cell lines, of which only $10(1.3 \%)$ represent complex soft-tissue sarcomas (another 38 represent Ewing sarcoma or primitive neuroectodermal tumor, rhabdomyosarcoma or osteosarcoma).

\section{Box 2}

\section{Identifying candidate driver alterations in noisy cancer genomes}

Over the course of their somatic evolution, cancer genomes can acquire an array of abnormalities. These alterations either confer a clonal growth advantage to the cell (driver) or are acquired stochastically, but are biologically neutral (passenger). The need to distinguish between these two alteration types in increasingly complex genomic data has driven the development of robust and statistically principled computational methodologies. Alteration-type-specific methods have focused in particular on DNA copy-number alterations (CNAs), one of the most common somatic genetic events not only in karyotypically complex sarcomas, but also in the genomes of epithelial cancers. Two such methods, Genomic Identification of Significant Targets in Cancer (GISTIC) 206 and $\mathrm{RAE}^{207}$, assign a statistical significance to candidate driver alterations emerging from a background of random, passenger abnormalities using their pattern of recurrence, amplitude, and extent, but also assign to individuals the set of CNAs they have undergone. The outputs of these computational methods can be used in studies of clinical associations, analyses of aberrant pathway activity, integrated with orthogonal data, or used to populate large-scale functional genetic screens (see main text). Alternatively, other methods, such as iCluster ${ }^{208}$ and Copy Number and Expression in Cancer (CONEXIC) ${ }^{209}$ identify putative driver alterations by integrating multiple highthroughput data types (such as expression and copy number).

There is, therefore, a pressing need to generate cell lines representative of diverse sarcoma types, mainly for the subtypes with complex karyotypes. The creation of a sarcoma cell line panel with cytogenetic and genomic profiles that mirror the diversity observed in their corresponding tumor types would represent a critical step in dissecting the influence of heterogeneity on variability of response to targeted therapies ${ }^{104}$. Such a panel could also drive genomics-guided functional genetics, either with arrayed or pooled loss-of-function RNAi screens ${ }^{105,106}$, or gain-of-function 'ORFeome' approaches ${ }^{107}$ (FIG. 3). 
Along these lines, we recently sought to functionally annotate the dedifferentiated liposarcoma genome by systematically knocking down genes altered by recurrent genomic amplification on 12q and elsewhere. With an arrayed loss-of-function shRNA screen, we determined which amplified genes are actually required for cell proliferation and survival ${ }^{74}$. We concentrated on dedifferentiated liposarcomas because the marked homogeneity of its genetic alterations compensates for the low number of cell lines available. Profiling of three dedifferentiated liposarcoma cell lines showed that this small panel captured a significant number of the molecular abnormalities observed in primary tumors. Using these validated cell lines, we identified several genes required for cancer cell viability, some of them potentially druggable. For instance, the hits included not only $C D K 4$ at $12 \mathrm{q} 14$, confirming its importance in this sarcoma, but also aurora kinase A ( $A U R K A$; at 20q13), specific inhibitors of which are currently in clinical trials ${ }^{108}$.

These studies also provided a setting where we could address an open question in cancer genetics, namely, whether focal genomic amplifications contain a single driver gene or, as recently suggested, multiple independent drivers ${ }^{109}$. We found evidence that $M D M 2$ and YEATS4, which are frequently co-amplified with each other (and nearly always in the same tumors with $C D K 4$ amplification) are both drivers ${ }^{74}$. MDM2 is a validated target in this disease, as drugs that inhibit the MDM2-p53 interaction induce apoptosis in dedifferentiated liposarcoma cell lines ${ }^{84,85}$. Therefore, these data support the concept of multiple driver genes in a single amplicon and hint at a more complex effect of genomic amplification on cancer phenotypes than previously understood. Furthermore, co-amplified genes may influence phenotypes unrelated to viability, so alternative assays are needed to test their role as oncogenes. Overall, this study design establishes a framework for the systematic genomic and functional genetic characterization of other rare cancers.

\section{In vivo models of sarcoma}

In addition to cell lines, several other types of models have been used for sarcoma and are likely similarly adaptable to in vivo functional genetics for target discovery. These include ex vivo cultures of tissue slices that preserve the original tumor microenvironment ${ }^{110}$ and low-passage short-term cultures ${ }^{111}, 112$, both of which are tractable surrogates of primary tumors. Nevertheless, to test novel targeted therapies, it is essential to develop in vivo models of sarcomas. Both subcutaneous and orthotopic xenografts (injecting sarcoma cell lines in immunocompromised mice) have been used to model human sarcomas but these model systems also have certain limitations. Some genetic abnormalities present in primary tumors will not be present or retained in the xenografts, and, conversely, serial passaging can introduce additional alterations not reflecting the primary tumors. Indeed, many treatments that initially showed promise in these models have not translated successfully to the clinic. To overcome these limitations, researchers are attempting to create panels of xenografts directly from primary tumor tissues representing several sarcoma subtypes ${ }^{113}$.

An alternative to xenografts is to genetically engineer animal models that reproduce the characteristics of human tumors, but that presents specific challenges. For mouse models of translocation-associated sarcomas, the challenge is to express the fusion oncogene in the correct lineage and development stage. For mouse models of complex karyotypes sarcomas, the challenge is expressing genuine alterations in an appropriate combination. For example, in leiomyosarcoma, the most prominent genetic alteration is chromosome 10 deletions affecting PTEN, but this may be a secondary alteration. Nonetheless, this was modeled by genetically inactivating Pten in smooth muscle cells of mice, which led to leiomyosarcomagenesis ${ }^{114}$. Another recent mouse model introduced oncogenic Kras and mutant $\operatorname{Trp} 53$ in the muscle of mice; these changes were sufficient to generate high-grade sarcomas with myofibroblastic differentiation ${ }^{115}$, but $K R A S$ is rarely mutated in human sarcomas. Sarcomas with simple karyotypes that have been successfully modeled are 
synovial sarcoma ${ }^{62,63}$, ARMS $^{116}$, myxoid liposarcoma ${ }^{117}$, and GIST ${ }^{118,119}$. Nevertheless, in one model of synovial sarcoma ${ }^{62}$, the SYT-SSX fusion oncogene was targeted to the myogenic lineage, a lineage inconsistent with conventional pathologic data on this sarcoma.

These and other sarcoma models may reveal the specific roles of genes altered in primary tumors and allow identification of secondary genetic or phenotypic events that are required for sarcoma progression and/or metastasis (FIG. 3). Engineered animal models may also be adapted to in vivo RNAi screens, as has been demonstrated in models of hepatocellular carcinoma and lymphoma ${ }^{120-122}$ (FIG. 3). This approach to exploring gene function would be especially powerful in soft-tissue sarcomas with complex genotypes and numerous chromosome aberrations. Genetically engineered animal models can also be used for diagnostic or prognostic biomarker discovery, drug testing, and drug resistance studies ${ }^{123}$. Indeed, the combination of sarcoma tumor profiles, sarcoma model systems that faithfully represent the alterations characteristic of their tumor type, and in vitro and in vivo functional genetics is a powerful approach to target discovery that is also being applied to other cancers by the National Cancer Institute's Cancer Target Discovery and Development Network ${ }^{124}$.

\section{Therapeutic avenues}

Despite the many advances in identifying genetic abnormalities in sarcoma and elucidating their function, cytotoxic chemotherapy remains the standard of care for most locally advanced and metastatic sarcomas. Yet, complete surgical resection is the best hope for cure, and few patients with unresectable disease are curable by cytotoxic chemotherapy. Indeed, today few specific genetic lesions in sarcoma are direct targets of therapy, unlike epithelial cancer types harboring mutations that confer sensitivity to targeted inhibitors ${ }^{125}$.

The exception among sarcomas is GIST, where the KIT kinase inhibitor imatinib achieves a partial response or stable disease in approximately $80 \%$ of patients with advanced or metastatic GIST, often within days, with some patients on therapy now for 10 years ${ }^{126}$. These responses to imatinib depend, however, on the specific site of mutation; tumors with activation loop mutations are generally insensitive. Response to imatinib has also been disappointing in patients with wild-type KIT and PDGFR $A$ genotypes, despite KIT pathway activation. These findings lend support to a genotype-driven paradigm of kinase inhibition. This paradigm may apply across tumors of diverse histologies that share addiction to a particular mutated kinase. For example, the recent success of Raf inhibitors in BRAFV600E mutant melanoma suggests that responses may be elicited in other tumor types with a dependence on oncogenic $\operatorname{Raf}^{127,128}$, a possible therapeutic option for the approximately $1 \%$ of adult GIST patients with BRAF-V600E mutation ${ }^{66}$.

GIST notwithstanding, other common sarcomas have shown very little sensitivity to existing tyrosine kinase inhibitors (TKIs) including leiomyosarcoma, high-grade undifferentiated pleomorphic sarcoma (formerly termed malignant fibrous histiocytoma) and welldifferentiated/dedifferentiated liposarcoma ${ }^{129}, 130$. Nevertheless, kinase-directed agents have produced responses in certain translocation-associated sarcomas ${ }^{130,131}$ (Table 2). Among these are responses to imatinib in dermatofibrosarcoma protuberans (DFSP) and giant-cell tumors of the tendon sheath with collagen Ia $1(C O L 1 A 1)$-platelet- derived growth factor- $\beta$ $(P D G F B)$ and collagen Iva3 (COL6A3)-colony-stimulating factor 1 (CSFI) fusions, respectively 132, 133, MET inhibitor responses in ASPS and clear-cell sarcomas with ASPLTFE3 and EWS-activating transcription factor 1 (ATF1) fusions, respectively 38, 134, 135, ALK inhibitor responses in inflammatory myofibroblastic tumors with ALK fusions ${ }^{136}$, and IGF1R antibody responses in Ewing sarcoma with EWS-FLI1 or EWS-ERG fusions ${ }^{137-139}$. Yet in none of these instances have clinical responses proven to be as durable as those observed in patients with GIST treated with imatinib or other TKIs. In addition, patients 
with Ewing sarcoma have an approximately $10-15 \%$ response rate to anti-IGF1R therapy, yet in these tumors and in angiosarcomas, preclinical evidence would have predicted a greater response rate $131,140,141$. The reason for this discrepancy is unknown, though perhaps only a fraction of patients have overtly IGF1R-dependent tumors, as indicated by high serum IGF1 levels, which has been observed in non-small cell lung cancer ${ }^{142}$.

Among some less common sarcoma subtypes, several targeted agents appear active (Table 2). In addition, the identification of moderately or highly prevalent genetic abnormalities in some subtypes has suggested new possibilities for therapy. VEGFR-directed therapies such as bevacizumab and sorafenib are associated with approximately $15 \%$ response rates in primary and radiation-induced angiosarcoma ${ }^{129}, 143$, perhaps associated most closely with $K D R$ mutation $^{73}$. ASPS tumors are sensitive to VEGF-directed therapy such as cediranib or sunitinib $^{144,145}$. On the basis of reduced expression of tuberin (TSC2), perivascular epithelial cell tumors (PEComas) and related conditions such as lymphangioleiomyomatosis and angiomyolipoma respond to mTOR inhibition ${ }^{146-148}$. As NF1 inactivation leads to aberrant MAPK and mTOR pathway activity ${ }^{149,150}$, the $N F 1$ mutations and genomic deletions recently observed in pleomorphic liposarcomas and myxofibrosarcomas ${ }^{74}$ may identify a broader range of patients who might respond to either RAF/MEK inhibitors or rapamycin and its analogs (rapalogues). In fact, deploying rapalogues in several complex subtypes could be justified on the basis of highly prevalent PTEN deletions, as in leiomyosarcoma ${ }^{114}$. However, preliminary results of a phase III study of the mTOR inhibitor ridoforolimus indicated that progression-free survival was extended by only 3.1 weeks after completion of cytotoxic chemotherapy compared with the control arm, suggesting limited utility of mTOR inhibitors in sarcoma patients not first selected on the basis of their genomic abnormalities. The finding of frequent PIK3CA mutations in myxoid/ round-cell liposarcoma ${ }^{74}$ (Table 1) suggests that at least this molecular subset of patients might benefit from PI3K inhibitors; this is currently being tested in clinical trials.

Finally, and while not strictly a targeted chemotherapeutic agent, trabectedin, a DNA minor groove-binding drug now approved in Europe for use in sarcomas, shows a substantial response rate in myxoid/round cell liposarcoma with FUS-CHOP and EWS-CHOPfusions and perhaps in additional translocation-associated sarcomas 151,152 . While the precise function of trabectedin in sarcomas is unclear, it appears to involve alterations in transcription downstream of histone and transcription factor binding ${ }^{153}$. Trabectedin sensitizes cancer cell lines to FAS-mediated cell death ${ }^{154}$ and sarcomas with intact nucleotide excision repair (NER) appear to be more sensitive to the drug than those with dysfunctional NER ${ }^{155}$.

\section{Acquired and adaptive resistance}

Malignancies, both epithelial and mesenchymal in origin, have remarkable similarity in their mechanisms of acquired and adaptive drug resistance. Resistance to TKIs is frequently acquired through reactivation of the oncogenic kinase through second-site mutations, as in KIT-mutant GIST ${ }^{156-158}$. For patients with metastatic disease, the median time to progression on first-line imatinib therapy is approximately 2 years. Here, the nature of secondary KIT mutations depends on the location of the primary KIT mutation. For instance, GIST harboring the more common and imatinib-sensitive KIT exon 11 mutation tend to become resistant by acquiring a second-site KIT mutation in exon 11 rather than in exon 9. Resistance to broadly based second-line KIT inhibitors (sunitinib), arising on average in $\sim 6$ months, can also develop through selection for double KIT-mutant resistant clones ${ }^{159}$. Another mechanism of resistance may involve alternative oncogenic pathways or rewiring of signaling networks, as experimental evidence suggests is the case for IGF1R inhibitors in rhabdomyosarcomas and Ewing sarcoma cell lines ${ }^{160,161}$. This adaptive 
resistance is consistent with the lack of $I G F 1 R$ mutations observed in cancer types where these therapies are active.

To circumvent these mechanisms of drug resistance, additional novel agents and strategies will be required. A strategy currently being used for imatinib-resistant chronic myelogenous leukemia is the development of second- or third-generation inhibitors with kinase-binding affinity or binding to sites other than the kinase domain itself ${ }^{162}$. The newer generation KIT inhibitors in preclinical development bind to the switch pocket domain of the protein, overcoming the resistance mediated by most combinations of KIT mutations observed in clinical samples ${ }^{163}$. However, the complexity of polyclonal resistance in imatinib-resistant GIST patients suggests that a single next-generation drug is unlikely to inhibit all mutant clones in a given patient, and broader therapeutic strategies need to be considered. Strategies being examined in clinical trials include drug combinations that block specific heterodimerization of receptor tyrosine kinases or multiple levels in a single signaling pathway. One such example is an inhibitor of CDC37, a protein that links KIT to the chaperone heat shock protein 90 (HSP90); this inhibitor may potentiate KIT degradation without the potential toxicity inherent in inhibiting too many HSP90 client proteins ${ }^{164-166}$. Other approaches warrant study, including polypharmacology, the simultaneous inhibition of multiple targets ${ }^{167}$.

\section{Transcriptional target genes as a therapeutic target}

Considering therapeutic strategies aimed at the aberrant transcriptional proteins driving translocation sarcomas, we note that transcription factors are considered poorly druggable because their protein-protein and protein-DNA interactions have historically been difficult to inhibit with small molecules. This view may be changing ${ }^{168,169}$, and in sarcoma, a notable example exists with a small molecule that disrupts a critical interaction of the EWSFLI1 protein with RNA helicase A in Ewing sarcoma ${ }^{170}$. Nevertheless, the most promising current approach to discovering therapeutic targets in translocation sarcomas is identifying targets of the chimeric transcription factor and focusing on those that encode known drug targets. The receptor tyrosine kinase MET has emerged as such a target in several sarcomas. $M E T$ is a direct transcriptional target of ASPL-TFE3 in ASPS ${ }^{38}$, and apparently also of PAX3-FOXO1 in ARMS 36,171 . In clear-cell sarcoma, EWS-ATF1 transactivates microphthalmia-associated transcription factor $(M I T F)$, which in turn directly activates MET transcription ${ }^{134,172}$. The fact that these fusion proteins upregulate MET has justified a phase II multi-institutional study of the MET inhibitor ARQ197 in patients with advanced clear cell sarcoma and ASPS. The transcriptional targets of the Ewing sarcoma fusion protein EWS-FLI1 appear to affect multiple pathways including Notch ${ }^{173}$, HedgehogGLI $^{174,175}$, Wnt- $\beta$-catenin ${ }^{176}$, transforming growth factor- $\beta$ (TGF $\left.\beta\right)^{177,}{ }^{178}$, and possibly IGF1R $^{179}$. The IGF1R pathway may be dysregulated by EWS-FLI1 at several levels, including $I G F 1$ upregulation and IGF binding protein 3 (IGFBP3) repression $138,180,181$. The IGF1R pathway is also transcriptionally upregulated by the PAX3-FOXO1 fusion in ARMS $^{34,35}$. These findings have in part provided the rationale for trials of IGF1R inhibitors in these sarcomas $141,182,183$. Finally, in myxoid/round cell liposarcoma, the FUS-CHOP fusion oncoprotein forms a complex with NFKBIZ at target promoters thereby upregulating nuclear factor- $\kappa \mathrm{B}(\mathrm{NF}-\kappa \mathrm{B})$ target genes ${ }^{184}$. Thus, NF- $\kappa \mathrm{B}$ pathway inhibition, which reduces the viability of myxoid liposarcoma cell lines ${ }^{185}$, may represent a new therapeutic option in this translocation sarcoma.

\section{Alternative therapeutic approaches}

The insensitivity of many sarcomas to existing systemic therapy is driving the exploration of agents aimed at new types of targets. Among these are HSP90 inhibitors, which have been studied in GISTs (but not in other sarcomas). Other novel targets include BCL2, 
phosphatases involved in feedback control of oncogenic pathways, and key mediators of epigenetic regulation including histone deacetylases, histone acetyltransferases, and DNA methyltransferases. Epigenetic approaches may lead to re-expression of pro-apoptotic molecules, rendering sarcomas sensitive to other agents, or itself induce apoptosis or senescence, although unknown at present. Similarly, cell cycle regulators including CDK4 and CDK6 ${ }^{186}$ have proven to be attractive but recalcitrant targets, while strategies to target components of the mitotic apparatus such as aurora kinases are actively under development. Targeting the p53-MDM2 pathway with nutlins is promising in tumors with MDM2 amplification $^{84}$ (predominantly well- and dedifferentiated liposarcomas). With an increasing array of agents to test against this rare group of cancers (FIG. 4), international-scale cooperative studies are paramount, as is ensuring that patients with sarcomas be included, along with more common cancers, in clinical trials of biologically relevant agents.

\section{Future directions}

The diagnosis and treatment of sarcoma patients is entering a period of rapid evolution. The dramatic drop in the cost of personal genome sequencing may alter the clinical and therapeutic course for sarcoma patients, as it is becoming technically possible to guide patient care by analysis of the patient's cancer and normal genome sequences and this may soon become practically feasible as well ${ }^{187}$. Over the next few years, the catalog of mutations that drive all but the least common diseases will become known, thanks to largescale efforts such as TCGA and the International Cancer Genome Consortium, as well as others. To prevent sarcomas from lagging behind epithelial cancers in target discovery, it will be critical that robust models of disease be developed to allow rapid functional annotation of the genetic abnormalities identified from both research and clinical sequencing.

\section{Acknowledgments}

We apologize to the many authors whose relevant work we were unable to cite here owing to space limitations. We thank N. Schultz for providing pathway expertise, C.D.M. Fletcher for critical reading, and M. Meyerson and C. Sander for advice and support. This work was supported in part by The Soft Tissue Sarcoma Program Project (P01 CA047179, S.S., M.L. and C.A.) and the SPORE in Soft Tissue Sarcoma (P50 CA140146-01, S.S., M.L., C.A. and B.S.T.).

\section{Glossary}

\section{Translocation}

Karyotypic
complexity

Myogenic

Second-generation sequencing
Structural rearrangement that juxtaposes distant genome sequences, resulting in aberrant gene expression or modified regulatory control of a gene (promoter substitution) or the formation of a fusion gene that encodes an aberrant, chimeric protein (gene fusion). Those pathognomonic translocations have more than diagnostic utility, rather they are the defining feature of the given tumor type

Tumors with a complex karyotype are those whose nuclear genome harbors numerical and structural abnormalities affecting multiple chromosomes

Originating in, or with expression specific to, muscular tissues

Sequencing methods and associated chemistries that sequence $>10^{6}$ nucleic acid fragments in parallel, producing short reads of $\sim 35-400$ bases. Used here synonymously with next-generation or massively parallel sequencing 


\author{
Neuroectodermal \\ Carney's triad \\ Carney-Stratakis
syndrome
}

Neurofibromatosis

Paraganglioma

Disease-specific survival

Chromothripsis

Episome

RNA interference

ORFeome

Response

Paired-end (matepaired)
Of the neuroectoderm, including neural crest and neural tube cell types

A rare syndrome in which there is a coexistence of three distinct tumor types: GIST, extra-adrenal paraganglioma, and pulmonary chondromas

Distinct from Carney's triad, and also referred to as the GISTparaganglioma dyad, a heritable syndrome in which familial mutations are associated with coexisting GIST and paraganglioma, but not pulmonary chondromas

Neurofibromatosis type I is an autosomal dominant genetic disorder in which tumors arise from nerve tissues and from all neural crest cell types

An uncommon neuroendocrine tumor arising from the sympathetic component of the autonomic nervous system and found predominantly in the abdomen, chest, or head and neck region

Patients with a given diagnosis who do not die of the specified disease in a defined period of time, which excludes patients who died from causes other than the studied disease

A neologism coined to describe a proposed single catastrophic remodeling of a chromosome and its accompanying punctuated model of somatic cancer evolution

An extra-chromosomal genetic entity, often circular, that can replicate autonomously

A technique for sequence-specific gene silencing in which small non-coding RNAs (principally microRNAs and small-interfering (si)RNAs) and associated regulatory complexes pair with complementary mRNA targets

A collection of cloned human protein-coding open reading frames suitable for stable expression via destination vectors in model systems (see Further information)

Used here as defined by RECIST criteria. The response evaluation criteria in solid tumors are guidelines developed to document a change in tumor burden and similarly monitor response to treatment during the clinical evaluation of cancer therapeutics

A technique whereby a library of genomic DNA or double-strand cDNA is created and circularized, and then short stretches (35$400 \mathrm{bp}$ ) are sequenced from either end of the cleaved product, but not the intervening variable-length fragment (from 200-500 bp up to $3-10 \mathrm{~kb}$ )

\section{References}

1. Fletcher, C.; Unni, K.; Mertens, F. Pathology and genetics of tumors of soft tissue and bone. International Agency for Research on Cancer Press; Lyon: 2002. 
2. Jemal A, Siegel R, Xu J, Ward E. Cancer statistics, 2010. CA Cancer J Clin. 2010; 60:277-300. [PubMed: 20610543]

3. Borden EC, et al. Soft tissue sarcomas of adults: state of the translational science. Clin Cancer Res. 2003; 9:1941-56. [PubMed: 12796356]

4. Helman LJ, Meltzer P. Mechanisms of sarcoma development. Nat Rev Cancer. 2003; 3:685-94. [PubMed: 12951587]

5. Mertens F, et al. Translocation-related sarcomas. Semin Oncol. 2009; 36:312-23. [PubMed: 19664492]

6. Mercado GE, Barr FG. Fusions involving PAX and FOX genes in the molecular pathogenesis of alveolar rhabdomyosarcoma: recent advances. Curr Mol Med. 2007; 7:47-61. [PubMed: 17311532]

7. Horvai AE, DeVries S, Roy R, O’Donnell RJ, Waldman F. Similarity in genetic alterations between paired well-differentiated and dedifferentiated components of dedifferentiated liposarcoma. Mod Pathol. 2009; 22:1477-88. [PubMed: 19734852]

8. Rosai J, et al. Combined morphologic and karyotypic study of 59 atypical lipomatous tumors. Evaluation of their relationship and differential diagnosis with other adipose tissue tumors (a report of the CHAMP Study Group). Am J Surg Pathol. 1996; 20:1182-9. [PubMed: 8827023]

9. Snyder EL, et al. c-Jun amplification and overexpression are oncogenic in liposarcoma but not always sufficient to inhibit the adipocytic differentiation programme. J Pathol. 2009; 218:292-300. [PubMed: 19449367]

10. Gregorian C, et al. PTEN dosage is essential for neurofibroma development and malignant transformation. Proc Natl Acad Sci U S A. 2009; 106:19479-84. [PubMed: 19846776]

11. Subramanian $S$, et al. Genome-wide transcriptome analyses reveal p53 inactivation mediated loss of miR-34a expression in malignant peripheral nerve sheath tumours. J Pathol. 2010; 220:58-70. [PubMed: 19890883]

12. van Beerendonk HM, et al. Molecular analysis of the INK4A/INK4A-ARF gene locus in conventional (central) chondrosarcomas and enchondromas: indication of an important gene for tumour progression. J Pathol. 2004; 202:359-66. [PubMed: 14991902]

13. Clark MA, Fisher C, Judson I, Thomas JM. Soft-tissue sarcomas in adults. N Engl J Med. 2005; 353:701-11. [PubMed: 16107623]

14. Mertens F, Panagopoulos I, Mandahl N. Genomic characteristics of soft tissue sarcomas. Virchows Arch. 2010; 456:129-39. [PubMed: 19189124]

15. Mani RS, Chinnaiyan AM. Triggers for genomic rearrangements: insights into genomic, cellular and environmental influences. Nat Rev Genet. 2010; 11:819-29. [PubMed: 21045868]

16. Novo FJ, Vizmanos JL. Chromosome translocations in cancer: computational evidence for the random generation of double-strand breaks. Trends Genet. 2006; 22:193-6. [PubMed: 16499992]

17. Soutoglou E, Misteli T. On the contribution of spatial genome organization to cancerous chromosome translocations. J Natl Cancer Inst Monogr. 2008:16-9. [PubMed: 18647996]

18. Hosaka T, et al. Translin binds to the sequences adjacent to the breakpoints of the TLS and CHOP genes in liposarcomas with translocation $\mathrm{t}(12 ; 6)$. Oncogene. 2000; 19:5821-5. [PubMed: 11126370]

19. Haffner MC, et al. Androgen-induced TOP2B-mediated double-strand breaks and prostate cancer gene rearrangements. Nat Genet. 2010; 42:668-75. [PubMed: 20601956]

20. Lin C, et al. Nuclear receptor-induced chromosomal proximity and DNA breaks underlie specific translocations in cancer. Cell. 2009; 139:1069-83. [PubMed: 19962179]

21. Mani RS, et al. Induced chromosomal proximity and gene fusions in prostate cancer. Science. 2009; 326:1230. [PubMed: 19933109]

22. Deraedt K, Debiec-Rychter M, Sciot R. Radiation-associated synovial sarcoma of the lung following radiotherapy for pulmonary metastasis of Wilms' tumour. Histopathology. 2006; 48:473-5. [PubMed: 16487376]

23. Egger JF, Coindre JM, Benhattar J, Coucke P, Guillou L. Radiation-associated synovial sarcoma: clinicopathologic and molecular analysis of two cases. Mod Pathol. 2002; 15:998-1004. [PubMed: 12218218] 
24. van de Rijn M, et al. Radiation-associated synovial sarcoma. Hum Pathol. 1997; 28:1325-8. [PubMed: 9385945]

25. Ohali A, et al. Different telomere maintenance mechanisms in alveolar and embryonal rhabdomyosarcoma. Genes Chromosomes Cancer. 2008; 47:965-70. [PubMed: 18663749]

26. Ulaner GA, et al. Divergent patterns of telomere maintenance mechanisms among human sarcomas: sharply contrasting prevalence of the alternative lengthening of telomeres mechanism in Ewing's sarcomas and osteosarcomas. Genes Chromosomes Cancer. 2004; 41:155-62. [PubMed: 15287028]

27. Lafferty-Whyte $\mathrm{K}$, et al. A gene expression signature classifying telomerase and ALT immortalization reveals an hTERT regulatory network and suggests a mesenchymal stem cell origin for ALT. Oncogene. 2009; 28:3765-74. [PubMed: 19684619]

28. Hsu JJ, et al. Werner syndrome gene variants in human sarcomas. Mol Carcinog. 2010; 49:166-74. [PubMed: 19824023]

29. Meyer S, et al. Rhabdomyosarcoma in Nijmegen breakage syndrome: strong association with perianal primary site. Cancer Genet Cytogenet. 2004; 154:169-74. [PubMed: 15474156]

30. Hicks MJ, Roth JR, Kozinetz CA, Wang LL. Clinicopathologic features of osteosarcoma in patients with Rothmund-Thomson syndrome. J Clin Oncol. 2007; 25:370-5. [PubMed: 17264332]

31. Werner SR, Prahalad AK, Yang J, Hock JM. RECQL4-deficient cells are hypersensitive to oxidative stress/damage: Insights for osteosarcoma prevalence and heterogeneity in RothmundThomson syndrome. Biochem Biophys Res Commun. 2006; 345:403-9. [PubMed: 16678792]

32. Stephens PJ, et al. Massive genomic rearrangement acquired in a single catastrophic event during cancer development. Cell. 2011; 144:27-40. This paper describes a new process of cancer genome evolution: the acquisition of multiple genomic abnormalities in a single catastrophic chromothripsis event, which is characteristic of a subset of osteosarcomas and chordomas. [PubMed: 21215367]

33. Mitelman F, Johansson B, Mertens F. The impact of translocations and gene fusions on cancer causation. Nat Rev Cancer. 2007; 7:233-45. [PubMed: 17361217]

34. Cao L, et al. Genome-wide identification of PAX3-FKHR binding sites in rhabdomyosarcoma reveals candidate target genes important for development and cancer. Cancer Res. 2010; 70:6497508. [PubMed: 20663909]

35. Ayalon D, Glaser T, Werner H. Transcriptional regulation of IGF-I receptor gene expression by the PAX3-FKHR oncoprotein. Growth Horm IGF Res. 2001; 11:289-97. [PubMed: 11735247]

36. Ginsberg JP, Davis RJ, Bennicelli JL, Nauta LE, Barr FG. Up-regulation of MET but not neural cell adhesion molecule expression by the PAX3-FKHR fusion protein in alveolar rhabdomyosarcoma. Cancer Res. 1998; 58:3542-6. [PubMed: 9721857]

37. Mercado GE, et al. Identification of PAX3-FKHR-regulated genes differentially expressed between alveolar and embryonal rhabdomyosarcoma: focus on MYCN as a biologically relevant target. Genes Chromosomes Cancer. 2008; 47:510-20. [PubMed: 18335505]

38. Tsuda M, et al. TFE3 fusions activate MET signaling by transcriptional up-regulation, defining another class of tumors as candidates for therapeutic MET inhibition. Cancer Res. 2007; 67:91929. [PubMed: 17283122]

39. Nagai M, Tsuda M, Saito T, Lae M, Ladanyi M. Functional properties of ASPL-TFE3 and identification of CYP17A1 and UPP1 as direct transcriptional targets. Proc Amer Assoc Cancer Res. 2005; 46:abstract 4518.

40. Guillon N, et al. The oncogenic EWS-FLI1 protein binds in vivo GGAA microsatellite sequences with potential transcriptional activation function. PLoS One. 2009; 4:e4932. [PubMed: 19305498]

41. Gangwal K, et al. Microsatellites as EWS/FLI response elements in Ewing's sarcoma. Proc Natl Acad Sci U S A. 2008; 105:10149-54. This paper illustrates the pattern of EWS-FLI1 binding to target genes; the over-representation of human promoters with GGAA microsatellites among bound genes and how these are used to regulate EWS-FLI1 target gene expression. [PubMed: 18626011]

42. Boeva V, et al. De novo motif identification improves the accuracy of predicting transcription factor binding sites in ChIP-Seq data analysis. Nucleic Acids Res. 2010; 38:e126. [PubMed: 20375099] 
43. Kovar H. Downstream EWS/FLI1 - upstream Ewing's sarcoma. Genome Med. 2010; 2:8. [PubMed: 20156317]

44. Graf T, Enver T. Forcing cells to change lineages. Nature. 2009; 462:587-94. [PubMed: 19956253]

45. Yamanaka S, Blau HM. Nuclear reprogramming to a pluripotent state by three approaches. Nature. 2010; 465:704-12. [PubMed: 20535199]

46. Lessnick SL, Dacwag CS, Golub TR. The Ewing's sarcoma oncoprotein EWS/FLI induces a p53dependent growth arrest in primary human fibroblasts. Cancer Cell. 2002; 1:393-401. [PubMed: 12086853]

47. Hu-Lieskovan S, et al. EWS-FLI1 fusion protein up-regulates critical genes in neural crest development and is responsible for the observed phenotype of Ewing's family of tumors. Cancer Res. 2005; 65:4633-44. [PubMed: 15930281]

48. Barr FG. Translocations, cancer and the puzzle of specificity. Nat Genet. 1998; 19:121-4. [PubMed: 9620766]

49. Lin PP, Wang Y, Lozano G. Mesenchymal stem cells and the origin of Ewing's sarcoma. Sarcoma. 2010; 2011

50. Kauer M, et al. A molecular function map of Ewing's sarcoma. PLoS One. 2009; 4:e5415. [PubMed: 19404404]

51. Tirode F, et al. Mesenchymal stem cell features of Ewing tumors. Cancer Cell. 2007; 11:421-9. This study describes a pattern of gene expression upon EWS-FLI1 silencing in Ewing sarcoma cells that suggest a mesenchymal stem cell origin, and demonstrates that these cells can be differentiated along a variety of lineages. [PubMed: 17482132]

52. Torchia EC, Jaishankar S, Baker SJ. Ewing tumor fusion proteins block the differentiation of pluripotent marrow stromal cells. Cancer Res. 2003; 63:3464-8. [PubMed: 12839926]

53. Li X, McGee-Lawrence ME, Decker M, Westendorf JJ. The Ewing's sarcoma fusion protein, EWS-FLI, binds Runx2 and blocks osteoblast differentiation. J Cell Biochem. 2010; 111:933-43. [PubMed: 20665663]

54. Riggi N, et al. EWS-FLI-1 modulates miRNA145 and SOX2 expression to initiate mesenchymal stem cell reprogramming toward Ewing sarcoma cancer stem cells. Genes Dev. 2010; 24:916-32. [PubMed: 20382729]

55. Riggi N, et al. EWS-FLI-1 expression triggers a Ewing's sarcoma initiation program in primary human mesenchymal stem cells. Cancer Res. 2008; 68:2176-85. [PubMed: 18381423]

56. Richter GH, et al. EZH2 is a mediator of EWS/FLI1 driven tumor growth and metastasis blocking endothelial and neuro-ectodermal differentiation. Proc Natl Acad Sci U S A. 2009; 106:5324-9. [PubMed: 19289832]

57. Fujino T, et al. Function of EWS-POU5F1 in sarcomagenesis and tumor cell maintenance. Am J Pathol. 2010; 176:1973-82. [PubMed: 20203285]

58. Yamaguchi S, et al. EWSR1 is fused to POU5F1 in a bone tumor with translocation $\mathrm{t}(6 ; 22)$ (p21;q12). Genes Chromosomes Cancer. 2005; 43:217-22. [PubMed: 15729702]

59. Antonescu CR, et al. EWSR1-POU5F1 fusion in soft tissue myoepithelial tumors. A molecular analysis of sixty-six cases, including soft tissue, bone, and visceral lesions, showing common involvement of the EWSR1 gene. Genes Chromosomes Cancer. 2010; 49:1114-24. [PubMed: 20815032]

60. Moller E, et al. POU5F1, encoding a key regulator of stem cell pluripotency, is fused to EWSR1 in hidradenoma of the skin and mucoepidermoid carcinoma of the salivary glands. J Pathol. 2008; 215:78-86. [PubMed: 18338330]

61. Naka N, et al. Synovial sarcoma is a stem cell malignancy. Stem Cells. 2010; 28:1119-31. [PubMed: 20518020]

62. Haldar M, Hancock JD, Coffin CM, Lessnick SL, Capecchi MR. A conditional mouse model of synovial sarcoma: insights into a myogenic origin. Cancer Cell. 2007; 11:375-88. [PubMed: 17418413]

63. Haldar M, Hedberg ML, Hockin MF, Capecchi MR. A CreER-based random induction strategy for modeling translocation-associated sarcomas in mice. Cancer Res. 2009; 69:3657-64. [PubMed: 19351831] 
64. Ren YX, et al. Mouse mesenchymal stem cells expressing PAX-FKHR form alveolar rhabdomyosarcomas by cooperating with secondary mutations. Cancer Res. 2008; 68:6587-97. [PubMed: 18701482]

65. Riggi N, et al. Expression of the FUS-CHOP fusion protein in primary mesenchymal progenitor cells gives rise to a model of myxoid liposarcoma. Cancer Res. 2006; 66:7016-23. [PubMed: 16849546]

66. Agaram NP, et al. Novel V600E BRAF mutations in imatinib-naive and imatinib-resistant gastrointestinal stromal tumors. Genes Chromosomes Cancer. 2008; 47:853-9. [PubMed: 18615679] References 66-68 were the first to describe the three most common molecular abnormalities of therapeutic potential in GIST, mutations in KIT, PDGFRA, and BRAF.

67. Heinrich MC, et al. PDGFRA activating mutations in gastrointestinal stromal tumors. Science. 2003; 299:708-10. [PubMed: 12522257] References 66-68 were the first to describe the three most common molecular abnormalities of therapeutic potential in GIST, mutations in KIT, $P D G F R A$, and $B R A F$.

68. Hirota $S$, et al. Gain-of-function mutations of c-kit in human gastrointestinal stromal tumors. Science. 1998; 279:577-80. [PubMed: 9438854] References 66-68 were the first to describe the three most common molecular abnormalities of therapeutic potential in GIST, mutations in KIT, $P D G F R A$, and $B R A F$.

69. Janeway KA, et al. Pediatric KIT wild-type and platelet-derived growth factor receptor alpha-wildtype gastrointestinal stromal tumors share KIT activation but not mechanisms of genetic progression with adult gastrointestinal stromal tumors. Cancer Res. 2007; 67:9084-8. [PubMed: 17909012]

70. Pasini B, et al. Clinical and molecular genetics of patients with the Carney-Stratakis syndrome and germline mutations of the genes coding for the succinate dehydrogenase subunits SDHB, SDHC, and SDHD. Eur J Hum Genet. 2008; 16:79-88. [PubMed: 17667967]

71. Maertens O, et al. Molecular pathogenesis of multiple gastrointestinal stromal tumors in NF1 patients. Hum Mol Genet. 2006; 15:1015-23. [PubMed: 16461335]

72. Mussi C, Schildhaus HU, Gronchi A, Wardelmann E, Hohenberger P. Therapeutic consequences from molecular biology for gastrointestinal stromal tumor patients affected by neurofibromatosis type 1. Clin Cancer Res. 2008; 14:4550-5. [PubMed: 18628470]

73. Antonescu CR, et al. KDR activating mutations in human angiosarcomas are sensitive to specific kinase inhibitors. Cancer Res. 2009; 69:7175-9. [PubMed: 19723655]

74. Barretina J, et al. Subtype-specific genomic alterations define new targets for soft-tissue sarcoma therapy. Nat Genet. 2010; 42:715-21. This paper describes the integrative genomic analysis of seven subtypes of sarcoma and pinpoints potential targets of therapy. [PubMed: 20601955]

75. Ito M, et al. Comprehensive mapping of p53 pathway alterations reveals an apparent role for both SNP309 and MDM2 amplification in sarcomagenesis. Clin Cancer Res. 2011; 17:416-26. [PubMed: 21159888]

76. Perot G, et al. Constant p53 pathway inactivation in a large series of soft tissue sarcomas with complex genetics. Am J Pathol. 2010; 177:2080-90. [PubMed: 20884963]

77. Huang HY, et al. Ewing sarcomas with p53 mutation or p16/p14ARF homozygous deletion: a highly lethal subset associated with poor chemoresponse. J Clin Oncol. 2005; 23:548-58. [PubMed: 15659501]

78. Beroukhim R, et al. The landscape of somatic copy-number alteration across human cancers. Nature. 2010; 463:899-905. [PubMed: 20164920]

79. Chibon F, et al. Validated prediction of clinical outcome in sarcomas and multiple types of cancer on the basis of a gene expression signature related to genome complexity. Nat Med. 2010; 16:7817. This study develops a prognostic signature of genes that reflects the complexity of sarcomas, predicts for metastasis outcome superior to conventional sarcoma grading, and can distinguish low- and high-risk patients in other malignancies. [PubMed: 20581836]

80. El-Rifai W, Sarlomo-Rikala M, Andersson LC, Knuutila S, Miettinen M. DNA sequence copy number changes in gastrointestinal stromal tumors: tumor progression and prognostic significance. Cancer Res. 2000; 60:3899-903. [PubMed: 10919666] 
81. Ylipaa A, et al. Integrative genomic characterization and a genomic staging system for gastrointestinal stromal tumors. Cancer. 2011; 117:380-9. [PubMed: 20818650]

82. Pedeutour F, et al. Structure of the supernumerary ring and giant rod chromosomes in adipose tissue tumors. Genes Chromosomes Cancer. 1999; 24:30-41. [PubMed: 9892106]

83. Sirvent N, et al. Detection of MDM2-CDK4 amplification by fluorescence in situ hybridization in 200 paraffin-embedded tumor samples: utility in diagnosing adipocytic lesions and comparison with immunohistochemistry and real-time PCR. Am J Surg Pathol. 2007; 31:1476-89. [PubMed: 17895748]

84. Muller CR, et al. Potential for treatment of liposarcomas with the MDM2 antagonist Nutlin-3A. Int J Cancer. 2007; 121:199-205. [PubMed: 17354236]

85. Singer S, et al. Gene expression profiling of liposarcoma identifies distinct biological types/ subtypes and potential therapeutic targets in well-differentiated and dedifferentiated liposarcoma. Cancer Res. 2007; 67:6626-36. [PubMed: 17638873]

86. Helias-Rodzewicz Z, Pedeutour F, Coindre JM, Terrier P, Aurias A. Selective elimination of amplified CDK4 sequences correlates with spontaneous adipocytic differentiation in liposarcoma. Genes Chromosomes Cancer. 2009; 48:943-52. [PubMed: 19626636]

87. Mayr C, Hemann MT, Bartel DP. Disrupting the pairing between let-7 and Hmga2 enhances oncogenic transformation. Science. 2007; 315:1576-9. [PubMed: 17322030]

88. Atiye J, et al. Gene amplifications in osteosarcoma-CGH microarray analysis. Genes Chromosomes Cancer. 2005; 42:158-63. [PubMed: 15540165]

89. Chibon F, et al. ASK1 (MAP3K5) as a potential therapeutic target in malignant fibrous histiocytomas with 12q14-q15 and 6q23 amplifications. Genes Chromosomes Cancer. 2004; 40:32-7. [PubMed: 15034865]

90. Heidenblad M, et al. Genomic profiling of bone and soft tissue tumors with supernumerary ring chromosomes using tiling resolution bacterial artificial chromosome microarrays. Oncogene. 2006; 25:7106-16. [PubMed: 16732325]

91. Mariani O, et al. JUN oncogene amplification and overexpression block adipocytic differentiation in highly aggressive sarcomas. Cancer Cell. 2007; 11:361-74. [PubMed: 17418412]

92. Helias-Rodzewicz Z, et al. YAP1 and VGLL3, encoding two cofactors of TEAD transcription factors, are amplified and overexpressed in a subset of soft tissue sarcomas. Genes Chromosomes Cancer. 2010; 49:1161-71. [PubMed: 20842732]

93. Idbaih A, et al. Myxoid malignant fibrous histiocytoma and pleomorphic liposarcoma share very similar genomic imbalances. Lab Invest. 2005; 85:176-81. [PubMed: 15702084]

94. Gibault L, et al. New insights in sarcoma oncogenesis: a comprehensive analysis of a large series of 160 soft tissue sarcomas with complex genomics. J Pathol. 2010; 223:64-71. [PubMed: 21125665]

95. Adamowicz M, et al. Frequent amplifications and abundant expression of TRIO, NKD2, and IRX2 in soft tissue sarcomas. Genes Chromosomes Cancer. 2006; 45:829-38. [PubMed: 16752383]

96. McDermott KM, et al. p16(INK4a) prevents centrosome dysfunction and genomic instability in primary cells. PLoS Biol. 2006; 4:e51. [PubMed: 16464125]

97. Shen WH, et al. Essential role for nuclear PTEN in maintaining chromosomal integrity. Cell. 2007; 128:157-70. [PubMed: 17218262]

98. Meza-Zepeda LA, et al. Array comparative genomic hybridization reveals distinct DNA copy number differences between gastrointestinal stromal tumors and leiomyosarcomas. Cancer Res. 2006; 66:8984-93. [PubMed: 16982739]

99. Beck AH, et al. Discovery of molecular subtypes in leiomyosarcoma through integrative molecular profiling. Oncogene. 2010; 29:845-54. [PubMed: 19901961]

100. Kimura Y, Morita T, Hayashi K, Miki T, Sobue K. Myocardin functions as an effective inducer of growth arrest and differentiation in human uterine leiomyosarcoma cells. Cancer Res. 2010; 70:501-11. [PubMed: 20068148]

101. Perot G, et al. Strong smooth muscle differentiation is dependent on myocardin gene amplification in most human retroperitoneal leiomyosarcomas. Cancer Res. 2009; 69:2269-78. [PubMed: 19276386] 
102. Garraway LA, Sellers WR. Lineage dependency and lineage-survival oncogenes in human cancer. Nat Rev Cancer. 2006; 6:593-602. [PubMed: 16862190]

103. Bignell GR, et al. Signatures of mutation and selection in the cancer genome. Nature. 2010; 463:893-8. [PubMed: 20164919]

104. Sharma SV, Haber DA, Settleman J. Cell line-based platforms to evaluate the therapeutic efficacy of candidate anticancer agents. Nat Rev Cancer. 2010; 10:241-53. [PubMed: 20300105]

105. Luo B, et al. Highly parallel identification of essential genes in cancer cells. Proc Natl Acad Sci U S A. 2008; 105:20380-5. [PubMed: 19091943]

106. Moffat J, et al. A lentiviral RNAi library for human and mouse genes applied to an arrayed viral high-content screen. Cell. 2006; 124:1283-98. [PubMed: 16564017]

107. Johannessen CM, et al. COT drives resistance to RAF inhibition through MAP kinase pathway reactivation. Nature. 2010; 468:968-72. [PubMed: 21107320]

108. Macarulla T, et al. Phase I study of the selective Aurora A kinase inhibitor MLN8054 in patients with advanced solid tumors: safety, pharmacokinetics, and pharmacodynamics. Mol Cancer Ther. 2010; 9:2844-52. [PubMed: 20724522]

109. Rui L, et al. Cooperative epigenetic modulation by cancer amplicon genes. Cancer Cell. 2010; 18:590-605. [PubMed: 21156283]

110. Vaira V, et al. Preclinical model of organotypic culture for pharmacodynamic profiling of human tumors. Proc Natl Acad Sci U S A. 2010; 107:8352-6. [PubMed: 20404174]

111. Martinez N, et al. Transcriptional signature of Ecteinascidin 743 (Yondelis, Trabectedin) in human sarcoma cells explanted from chemo-naive patients. Mol Cancer Ther. 2005; 4:814-23. [PubMed: 15897246]

112. Peng T, et al. An experimental model for the study of well-differentiated and dedifferentiated liposarcoma; deregulation of targetable tyrosine kinase receptors. Lab Invest. 2011; 91:392-403. [PubMed: 21060307]

113. Frapolli R, et al. Novel models of myxoid liposarcoma xenografts mimicking the biological and pharmacologic features of human tumors. Clin Cancer Res. 2010; 16:4958-67. [PubMed: 20732964]

114. Hernando E, et al. The AKT-mTOR pathway plays a critical role in the development of leiomyosarcomas. Nat Med. 2007; 13:748-53. [PubMed: 17496901]

115. Kirsch DG, et al. A spatially and temporally restricted mouse model of soft tissue sarcoma. Nat Med. 2007; 13:992-7. [PubMed: 17676052]

116. Keller C, et al. Alveolar rhabdomyosarcomas in conditional Pax3:Fkhr mice: cooperativity of Ink4a/ARF and Trp53 loss of function. Genes Dev. 2004; 18:2614-26. [PubMed: 15489287]

117. Perez-Losada J, et al. The chimeric FUS/TLS-CHOP fusion protein specifically induces liposarcomas in transgenic mice. Oncogene. 2000; 19:2413-22. [PubMed: 10828883]

118. Sommer G, et al. Gastrointestinal stromal tumors in a mouse model by targeted mutation of the Kit receptor tyrosine kinase. Proc Natl Acad Sci U S A. 2003; 100:6706-11. [PubMed: 12754375]

119. Rubin BP, et al. A knock-in mouse model of gastrointestinal stromal tumor harboring kit K641E. Cancer Res. 2005; 65:6631-9. [PubMed: 16061643]

120. Zender L, et al. An oncogenomics-based in vivo RNAi screen identifies tumor suppressors in liver cancer. Cell. 2008; 135:852-64. [PubMed: 19012953] References 120-121 describe the application of RNAi screening to in vivo models of cancer for functional annotation of cancer genomes.

121. Bric A, et al. Functional identification of tumor-suppressor genes through an in vivo RNA interference screen in a mouse lymphoma model. Cancer Cell. 2009; 16:324-35. [PubMed: 19800577] References 120-121 describe the application of RNAi screening to in vivo models of cancer for functional annotation of cancer genomes.

122. Meacham CE, Ho EE, Dubrovsky E, Gertler FB, Hemann MT. In vivo RNAi screening identifies regulators of actin dynamics as key determinants of lymphoma progression. Nat Genet. 2009; 41:1133-7. [PubMed: 19783987]

123. Dodd RD, Mito JK, Kirsch DG. Animal models of soft-tissue sarcoma. Dis Model Mech. 2010; 3:557-66. [PubMed: 20713645] 
124. Schreiber SL, et al. Towards patient-based cancer therapeutics. Nat Biotechnol. 2010; 28:904-6. [PubMed: 20829823]

125. Pao W, Chmielecki J. Rational, biologically based treatment of EGFR-mutant non-small-cell lung cancer. Nat Rev Cancer. 2010; 10:760-74. [PubMed: 20966921]

126. Heinrich MC, et al. Kinase mutations and imatinib response in patients with metastatic gastrointestinal stromal tumor. J Clin Oncol. 2003; 21:4342-9. [PubMed: 14645423]

127. Bollag G, et al. Clinical efficacy of a RAF inhibitor needs broad target blockade in BRAF-mutant melanoma. Nature. 2010; 467:596-9. [PubMed: 20823850]

128. Flaherty KT, et al. Inhibition of mutated, activated BRAF in metastatic melanoma. N Engl J Med. 2010; 363:809-819. [PubMed: 20818844]

129. Maki RG, et al. Phase II study of sorafenib in patients with metastatic or recurrent sarcomas. J Clin Oncol. 2009; 27:3133-40. [PubMed: 19451436]

130. Sleijfer S, et al. Pazopanib, a multikinase angiogenesis inhibitor, in patients with relapsed or refractory advanced soft tissue sarcoma: a phase II study from the European organisation for research and treatment of cancer-soft tissue and bone sarcoma group (EORTC study 62043). J Clin Oncol. 2009; 27:3126-32. [PubMed: 19451427]

131. Pappo A, et al. Activity of R1507, a monoclonal antibody to the insulin-like growth factor-1 receptor, in patients with recurrent or refractory Ewing's sarcoma family of tumors: Results of a phase II SARC study. J Clin Oncol. 2010; 28 (suppl; abstr 10000).

132. Maki RG, Awan RA, Dixon RH, Jhanwar S, Antonescu CR. Differential sensitivity to imatinib of 2 patients with metastatic sarcoma arising from dermatofibrosarcoma protuberans. Int J Cancer. 2002; 100:623-6. [PubMed: 12209598]

133. McArthur GA, et al. Molecular and clinical analysis of locally advanced dermatofibrosarcoma protuberans treated with imatinib: Imatinib Target Exploration Consortium Study B2225. J Clin Oncol. 2005; 23:866-73. [PubMed: 15681532]

134. Davis IJ, et al. Oncogenic MITF dysregulation in clear cell sarcoma: defining the MiT family of human cancers. Cancer Cell. 2006; 9:473-84. [PubMed: 16766266]

135. McGill GG, Haq R, Nishimura EK, Fisher DE. c-Met expression is regulated by Mitf in the melanocyte lineage. J Biol Chem. 2006; 281:10365-73. [PubMed: 16455654]

136. Mosse YP, Wood A, Maris JM. Inhibition of ALK signaling for cancer therapy. Clin Cancer Res. 2009; 15:5609-14. [PubMed: 19737948]

137. Kolb EA, Gorlick R. Development of IGF-IR inhibitors in pediatric sarcomas. Curr Oncol Rep. 2009; 11:307-13. [PubMed: 19508836]

138. Scotlandi K, et al. Insulin-like growth factor I receptor-mediated circuit in Ewing's sarcoma/ peripheral neuroectodermal tumor: a possible therapeutic target. Cancer Res. 1996; 56:4570-4. [PubMed: 8840962]

139. Tolcher AW, et al. Phase I, pharmacokinetic, and pharmacodynamic study of AMG 479, a fully human monoclonal antibody to insulin-like growth factor receptor 1. J Clin Oncol. 2009; 27:5800-7. [PubMed: 19786654]

140. Kurzrock R, et al. A phase I study of weekly R1507, a human monoclonal antibody insulin-like growth factor-I receptor antagonist, in patients with advanced solid tumors. Clin Cancer Res. 2010; 16:2458-65. [PubMed: 20371689]

141. Olmos D, et al. Safety, pharmacokinetics, and preliminary activity of the anti-IGF-1R antibody figitumumab (CP-751,871) in patients with sarcoma and Ewing's sarcoma: a phase 1 expansion cohort study. Lancet Oncol. 2010; 11:129-35. [PubMed: 20036194]

142. Gualberto A, et al. Pre-treatment levels of circulating free IGF-1 identify NSCLC patients who derive clinical benefit from figitumumab. Br J Cancer. 2011; 104:68-74. [PubMed: 21102589]

143. Park MS, Ravi V, Araujo DM. Inhibiting the VEGF-VEGFR pathway in angiosarcoma, epithelioid hemangioendothelioma, and hemangiopericytoma/solitary fibrous tumor. Curr Opin Oncol. 2010; 22:351-5. [PubMed: 20485168]

144. Gardner K, Leahy M, Alvarez-Gutierrez M, Judson I, Scurr M. Activity of the VEGFR/KIT tyrosine kinase inhibitor cediranib (AZD2171) in alveolar soft part sarcoma. Proc Connective Tissue Oncology Society. 2008:abstract 34936. 
145. Stacchiotti S, et al. Response to sunitinib malate in advanced alveolar soft part sarcoma. Clin Cancer Res. 2009; 15:1096-104. [PubMed: 19188185]

146. Bissler JJ, et al. Sirolimus for angiomyolipoma in tuberous sclerosis complex or lymphangioleiomyomatosis. N Engl J Med. 2008; 358:140-51. [PubMed: 18184959]

147. Taille C, Debray MP, Crestani B. Sirolimus treatment for pulmonary lymphangioleiomyomatosis. Ann Intern Med. 2007; 146:687-8. [PubMed: 17470843]

148. Wagner AJ, et al. Clinical activity of mTOR inhibition with sirolimus in malignant perivascular epithelioid cell tumors: targeting the pathogenic activation of mTORC1 in tumors. J Clin Oncol. 2010; 28:835-40. [PubMed: 20048174]

149. Cichowski K, Santiago S, Jardim M, Johnson BW, Jacks T. Dynamic regulation of the Ras pathway via proteolysis of the NF1 tumor suppressor. Genes Dev. 2003; 17:449-54. [PubMed: 12600938]

150. Johannessen CM, et al. The NF1 tumor suppressor critically regulates TSC2 and mTOR. Proc Natl Acad Sci U S A. 2005; 102:8573-8. [PubMed: 15937108]

151. Forni C, et al. Trabectedin (ET-743) promotes differentiation in myxoid liposarcoma tumors. Mol Cancer Ther. 2009; 8:449-57. [PubMed: 19190116]

152. Grosso F, et al. Trabectedin in myxoid liposarcomas (MLS): a long-term analysis of a singleinstitution series. Ann Oncol. 2009; 20:1439-44. [PubMed: 19465423]

153. Minuzzo M, et al. Selective effects of the anticancer drug Yondelis (ET-743) on cell-cycle promoters. Mol Pharmacol. 2005; 68:1496-503. [PubMed: 15961672]

154. Martinez-Serra J, et al. Yondelis(R) (ET-743, Trabectedin) sensitizes cancer cell lines to CD95mediated cell death: New molecular insight into the mechanism of action. Eur J Pharmacol. 2011; 658:57-64. [PubMed: 21371453]

155. Schoffski P, et al. Predictive impact of DNA repair functionality on clinical outcome of advanced sarcoma patients treated with trabectedin: A retrospective multicentric study. Eur J Cancer. 2011; 47:1006-12. [PubMed: 21376569]

156. Gramza AW, Corless CL, Heinrich MC. Resistance to tyrosine kinase inhibitors in gastrointestinal stromal tumors. Clin Cancer Res. 2009; 15:7510-7518. [PubMed: 20008851]

157. Heinrich MC, et al. Molecular correlates of imatinib resistance in gastrointestinal stromal tumors. J Clin Oncol. 2006; 24:4764-74. [PubMed: 16954519]

158. Antonescu CR, et al. Acquired resistance to imatinib in gastrointestinal stromal tumor occurs through secondary gene mutation. Clin Cancer Res. 2005; 11:4182-90. [PubMed: 15930355]

159. Guo T, et al. Mechanisms of sunitinib resistance in gastrointestinal stromal tumors harboring KITAY502-3ins mutation: an in vitro mutagenesis screen for drug resistance. Clin Cancer Res. 2009; 15:6862-70. [PubMed: 19861442]

160. Huang F, et al. The mechanisms of differential sensitivity to an insulin-like growth factor-1 receptor inhibitor (BMS-536924) and rationale for combining with EGFR/HER2 inhibitors. Cancer Res. 2009; 69:161-70. [PubMed: 19117999]

161. Potratz JC, et al. Synthetic lethality screens reveal RPS6 and MST1R as modifiers of insulin-like growth factor-1 receptor inhibitor activity in childhood sarcomas. Cancer Res. 2010; 70:8770-81. [PubMed: 20959493]

162. Kantarjian H, et al. Dasatinib versus imatinib in newly diagnosed chronic-phase chronic myeloid leukemia. N Engl J Med. 2010; 362:2260-70. [PubMed: 20525995]

163. Heinrich MC, et al. In vitro activity of novel KIT/PDGFRA switch pocket kinase inhibitors against mutations associated with drug-resistant GI stromal tumors. J Clin Oncol. 2010; 28 (suppl; abstr 10007).

164. Smith JR, Workman P. Targeting CDC37: an alternative, kinase-directed strategy for disruption of oncogenic chaperoning. Cell Cycle. 2009; 8:362-72. [PubMed: 19177013]

165. Cutforth T, Rubin GM. Mutations in Hsp83 and cdc37 impair signaling by the sevenless receptor tyrosine kinase in Drosophila. Cell. 1994; 77:1027-36. [PubMed: 8020093]

166. Stepanova L, Leng X, Parker SB, Harper JW. Mammalian p50Cdc37 is a protein kinase-targeting subunit of Hsp90 that binds and stabilizes Cdk4. Genes Dev. 1996; 10:1491-502. [PubMed: 8666233] 
167. Knight ZA, Lin H, Shokat KM. Targeting the cancer kinome through polypharmacology. Nat Rev Cancer. 2010; 10:130-7. [PubMed: 20094047]

168. Dunker AK, Uversky VN. Drugs for 'protein clouds': targeting intrinsically disordered transcription factors. Curr Opin Pharmacol. 2010; 10:782-8. [PubMed: 20889377]

169. Koehler AN. A complex task? Direct modulation of transcription factors with small molecules. Curr Opin Chem Biol. 2010; 14:331-40. [PubMed: 20395165]

170. Erkizan HV, et al. A small molecule blocking oncogenic protein EWS-FLI1 interaction with RNA helicase A inhibits growth of Ewing's sarcoma. Nat Med. 2009; 15:750-6. The paper describes the first small-molecule inhibitor that directly targets an oncogenic chimeric transcription protein in sarcomas. [PubMed: 19584866]

171. Taulli R, et al. Validation of met as a therapeutic target in alveolar and embryonal rhabdomyosarcoma. Cancer Res. 2006; 66:4742-9. [PubMed: 16651427]

172. Davis IJ, et al. Identification of the receptor tyrosine kinase c-Met and its ligand, hepatocyte growth factor, as therapeutic targets in clear cell sarcoma. Cancer Res. 2010; 70:639-45. [PubMed: 20068147]

173. Ban J, et al. EWS-FLI1 suppresses NOTCH-activated p53 in Ewing's sarcoma. Cancer Res. 2008; 68:7100-9. [PubMed: 18757425]

174. Beauchamp E, et al. GLI1 is a direct transcriptional target of EWS-FLI1 oncoprotein. J Biol Chem. 2009; 284:9074-82. [PubMed: 19189974]

175. Zwerner JP, et al. The EWS/FLI1 oncogenic transcription factor deregulates GLI1. Oncogene. 2008; 27:3282-91. [PubMed: 18084326]

176. Navarro D, Agra N, Pestana A, Alonso J, Gonzalez-Sancho JM. The EWS/FLI1 oncogenic protein inhibits expression of the Wnt inhibitor DICKKOPF-1 gene and antagonizes betacatenin/TCF-mediated transcription. Carcinogenesis. 2010; 31:394-401. [PubMed: 20019092]

177. Hahm KB. Repression of the gene encoding the TGF-beta type II receptor is a major target of the EWS-FLI1 oncoprotein. Nat Genet. 1999; 23:481. [PubMed: 10581042]

178. Im YH, et al. EWS-FLI1, EWS-ERG, and EWS-ETV1 oncoproteins of Ewing tumor family all suppress transcription of transforming growth factor beta type II receptor gene. Cancer Res. 2000; 60:1536-40. [PubMed: 10749119]

179. Herrero-Martin D, et al. Stable interference of EWS-FLI1 in an Ewing sarcoma cell line impairs IGF-1/IGF-1R signalling and reveals TOPK as a new target. Br J Cancer. 2009; 101:80-90. [PubMed: 19491900]

180. Cironi L, et al. IGF1 is a common target gene of Ewing's sarcoma fusion proteins in mesenchymal progenitor cells. PLoS One. 2008; 3:e2634. [PubMed: 18648544]

181. Prieur A, Tirode F, Cohen P, Delattre O. EWS/FLI-1 silencing and gene profiling of Ewing cells reveal downstream oncogenic pathways and a crucial role for repression of insulin-like growth factor binding protein 3. Mol Cell Biol. 2004; 24:7275-83. [PubMed: 15282325]

182. Maki RG. Small is beautiful: insulin-like growth factors and their role in growth, development, and cancer. J Clin Oncol. 2010; 28:4985-95. [PubMed: 20975071]

183. Toretsky JA, Gorlick R. IGF-1R targeted treatment of sarcoma. Lancet Oncol. 2010; 11:105-6. [PubMed: 20036195]

184. Goransson M, et al. The myxoid liposarcoma FUS-DDIT3 fusion oncoprotein deregulates NFkappaB target genes by interaction with NFKBIZ. Oncogene. 2009; 28:270-8. [PubMed: 18850010]

185. Willems SM, et al. Kinome profiling of myxoid liposarcoma reveals NF-kappaB-pathway kinase activity and casein kinase II inhibition as a potential treatment option. Mol Cancer. 2010; 9:257. [PubMed: 20863376]

186. Malumbres M, Barbacid M. Cell cycle, CDKs and cancer: a changing paradigm. Nat Rev Cancer. 2009; 9:153-66. [PubMed: 19238148]

187. Ashley EA, et al. Clinical assessment incorporating a personal genome. Lancet. 2010; 375:152535. This paper illustrates the possibility of translating an individual's genome sequence, inferred risk, and familial context into clinical utility. [PubMed: 20435227]

188. Chi P, et al. ETV1 is a lineage survival factor that cooperates with KIT in gastrointestinal stromal tumours. Nature. 2010; 467:849-53. This paper describes the cellular context-dependent 
cooperation of the ETS family transcription factor ETV1 with mutant KIT in Interstitial cells of Cajal, the cells of origin of GISTs. [PubMed: 20927104]

189. Errani C, et al. A novel WWTR1-CAMTA1 gene fusion is a consistent abnormality in epithelioid hemangioendothelioma of different anatomic sites. Genes Chromosomes Cancer. 201110.1002/ gcc. 20886

190. Wang, L., et al. Identification of a novel, recurrent HEY1-NCOA2 fusion in mesenchymal chondrosarcoma based on a genome-wide screen of exon-level expression data. Submitted

191. Amary MF, et al. IDH1 and IDH2 mutations are frequent events in central chondrosarcoma and central and periosteal chondromas but not in other mesenchymal tumours. J Pathol. 2011 Somatic mutations in isocitrate dehydrogenase 1 (IDH1) and IDH2, first discovered in gliomas and then in acute myeloid leukaemias, are reported here for the first time in a sarcoma. Given that mutant IDH alleles have been causally associated with global DNA hypermethylation, this raises the possibility of widespread epigenetic changes in a subset of chondrosarcomas. 10.1002/path.2913

192. Blay JY, El Sayadi H, Thiesse P, Garret J, Ray-Coquard I. Complete response to imatinib in relapsing pigmented villonodular synovitis/tenosynovial giant cell tumor (PVNS/TGCT). Ann Oncol. 2008; 19:821-2. [PubMed: 18296418]

193. Antonescu CR. The GIST paradigm: lessons for other kinase-driven cancers. J Pathol. 2011; 223:251-61. [PubMed: 21125679]

194. Skubitz KM, Manivel JC, Clohisy DR, Frolich JW. Response of imatinib-resistant extraabdominal aggressive fibromatosis to sunitinib: case report and review of the literature on response to tyrosine kinase inhibitors. Cancer Chemother Pharmacol. 2009; 64:635-40. [PubMed: 19404642]

195. Gounder M, et al. Activity of sorafenib against desmoid tumor/deep fibromatoses. Clin Cancer Res. 2011 Epub ahead of print.

196. Thomas D, et al. Denosumab in patients with giant-cell tumour of bone: an open-label, phase 2 study. Lancet Oncol. 2010; 11:275-80. [PubMed: 20149736]

197. Butrynski JE, et al. Crizotinib in ALK-rearranged inflammatory myofibroblastic tumor. N Engl J Med. 2010; 363:1727-33. [PubMed: 20979472]

198. Goldberg J, et al. Preliminary results from a phase II study of ARQ 197 in patients with microphthalmia transcription factor family (MiT)-associated tumors. J Clin Oncol. 2009; 27 (suppl; abstr 10502).

199. Quek R, et al. Combination mTOR and IGF-1R inhibition: phase I trial of everolimus and figitumumab in patients with advanced sarcomas and other solid tumors. Clin Cancer Res. 2011; 17:871-9. [PubMed: 21177764]

200. Stacchiotti S, et al. Sunitinib malate and figitumumab in solitary fibrous tumor: patterns and molecular bases of tumor response. Mol Cancer Ther. 2010; 9:1286-97. [PubMed: 20457621]

201. Agulnik M, et al. An open-label multicenter phase II study of bevacizumab for the treatment of angiosarcoma. J Clin Oncol. 2009; 27 (suppl; abstr 10522).

202. Meyerson M, Gabriel S, Getz G. Advances in understanding cancer genomes through secondgeneration sequencing. Nat Rev Genet. 2010; 11:685-96. [PubMed: 20847746]

203. Maher CA, et al. Transcriptome sequencing to detect gene fusions in cancer. Nature. 2009; 458:97-101. [PubMed: 19136943]

204. Maher CA, et al. Chimeric transcript discovery by paired-end transcriptome sequencing. Proc Natl Acad Sci U S A. 2009; 106:12353-8. [PubMed: 19592507]

205. Post SM, et al. A high-frequency regulatory polymorphism in the $\mathrm{p} 53$ pathway accelerates tumor development. Cancer Cell. 2010; 18:220-30. [PubMed: 20832750]

206. Beroukhim R, et al. Assessing the significance of chromosomal aberrations in cancer: methodology and application to glioma. Proc Natl Acad Sci U S A. 2007; 104:20007-12. [PubMed: 18077431]

207. Taylor BS, et al. Functional copy-number alterations in cancer. PLoS ONE. 2008; 3:e3179. [PubMed: 18784837]

208. Shen R, Olshen AB, Ladanyi M. Integrative clustering of multiple genomic data types using a joint latent variable model with application to breast and lung cancer subtype analysis. Bioinformatics. 2009; 25:2906-12. [PubMed: 19759197] 
209. Akavia UD, et al. An integrated approach to uncover drivers of cancer. Cell. 2010; 143:1005-17. [PubMed: 21129771]

\section{Biographies}

\section{BARRY S. TAYLOR}

Barry S. Taylor, Ph.D., is the David H. Koch Fellow in cancer genomics at Memorial SloanKettering Cancer Center and a visiting scientist at the Helen Diller Family Comprehensive Cancer Center at the University of California, San Francisco. His research uses statistical, integrative genomic, and functional genetic approaches for cancer genome discovery.

\section{JORDI BARRETINA}

Jordi Barretina, Ph.D., is a research scientist in the Cancer Program at the Broad Institute. $\mathrm{He}$ is interested in applying genomic and functional tools to the analysis of cancer genomes with a particular focus on translational research. He currently leads the Cancer Cell Line Encyclopedia (CCLE) project, which is conducting detailed genetic and pharmacologic characterization of cancer cell lines to link therapeutic vulnerabilities to genomic patterns and translate cell line genomics into patient stratification in genotype-driven clinical trials.

\section{ROBERT G. MAKI}

Robert G. Maki, M.D., Ph.D, is the leader of the sarcoma program and pediatric hematology-oncology program at Mount Sinai Medical Center, New York. He is interested in and has been involved in numerous translational studies involving a variety of sarcoma subtypes, and is actively seeking both novel agents and new clinical trial designs to accelerate the use of new agents in adult and pediatric sarcoma patients.

\section{CRISTINA R. ANTONESCU}

Cristina R. Antonescu, M.D. is an Associate Attending Pathologist in the Department of Pathology at Memorial Sloan-Kettering Cancer Center. She is a sarcoma pathologist and her research focuses on characterizing novel molecular markers for diagnosis and treatment of gastrointestinal stromal tumors and angiosarcoma.

\section{SAMUEL SINGER}

Samuel Singer, M.D., FACS, is an Attending Surgical Oncologist and the Chief of the Gastric and Mixed Tumor Service at Memorial Sloan-Kettering Cancer Center, where he is principal investigator of the Soft Tissue Sarcoma Program Project and the SPORE in soft tissue sarcoma. His research focuses on an integrated molecular, genetic, and biochemical analysis of soft-tissue sarcoma. Dr. Singer also maintains a large clinical practice focused on patients with soft tissue sarcoma.

\section{MARC LADANYI}

Marc Ladanyi, M.D. holds the William Ruane Chair in Molecular Oncology at MSKCC where he is Attending Pathologist in the Molecular Diagnostics Service of the Department of Pathology and Member in the Human Oncology and Pathogenesis Program. He is a molecular pathologist whose research laboratory works on the genomics and molecular pathogenesis of sarcomas and thoracic malignancies. He also co-directs the Cancer Genome Atlas (TCGA) group at MSKCC, part of the NCI's TCGA project network. 


\section{At a glance}

- Human sarcomas are uncommon malignancies that arise from mesenchymal cell types, have varied genetic origins, and are clinically heterogeneous. Many sarcomas arise de novo, driven by a single genetic abnormality, while some are progressive and harbor complex genomes.

- Three core and context-dependent molecular mechanisms drive sarcomagenesis: dysregulation of gene expression by aberrant, chimeric transcription factors generated by specific gene fusions in translocation-associated sarcomas, somatic mutations affecting key signaling pathways, and DNA copy number abnormalities.

- Novel genomic findings from diverse approaches in sarcoma are identifying point mutations that co-occur with translocations, lineage-specific oncogenes, chromosomal remodeling events, and both genomic alterations and mutations that alter canonical signaling and differentiation pathways.

- As integrative genomics and massively parallel sequencing increase the pace of discovery for the most common lesions in all but the rarest sarcomas, this necessitates renewed focus on developing in vitro and in vivo sarcoma models for accompanying target discovery and functional annotation of sarcoma genomes with genomics-guided functional genetics.

- While conventional modalities predominate in sarcoma treatment, new approaches to target aberrant signaling with specific therapies, overcome acquired resistance, and target unconventional pathways are evolving rapidly. 


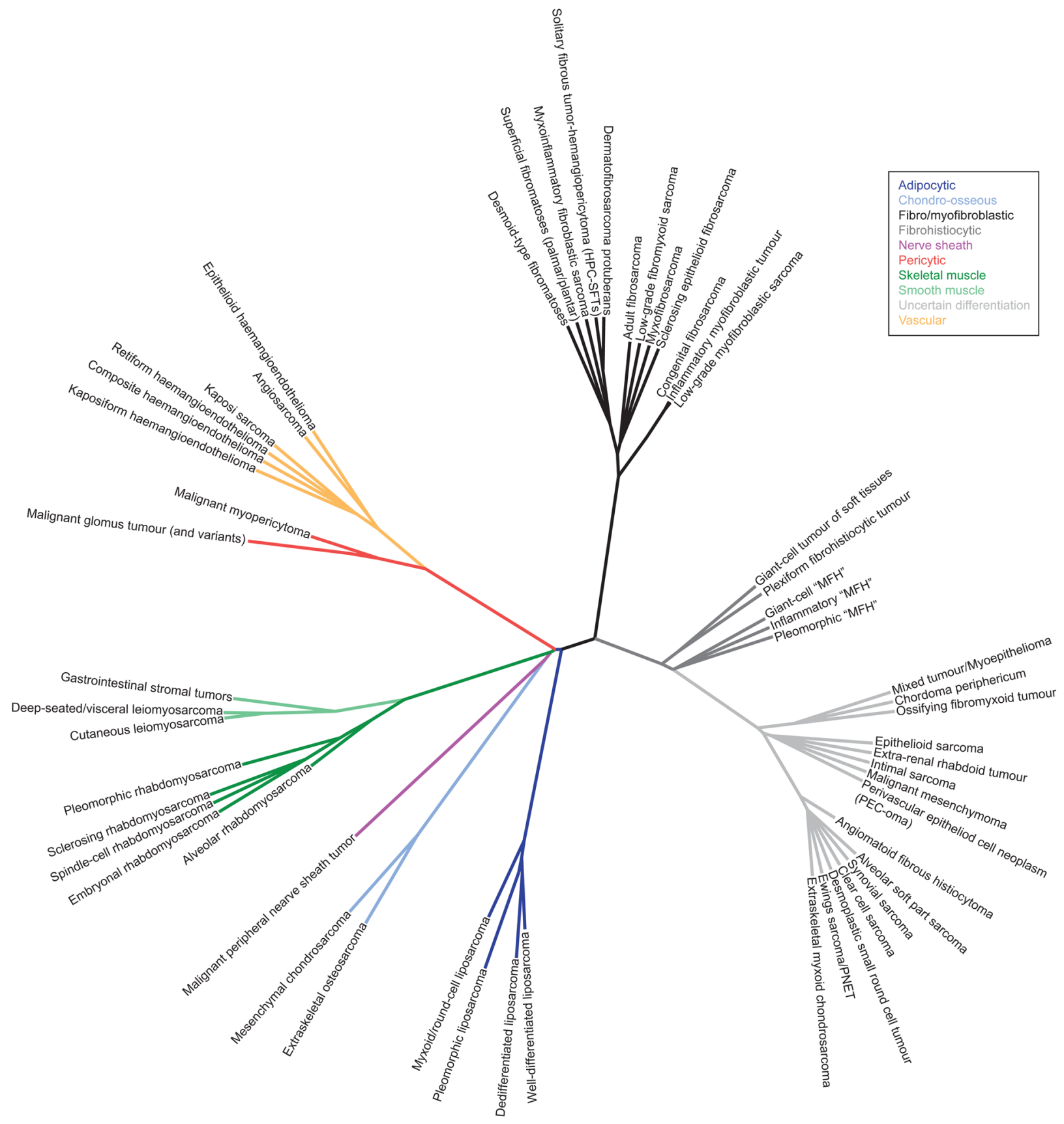

Figure 1. Taxonomy of soft tissue sarcoma

This unrooted phylogeny shows $\sim 60$ sarcoma subtypes as originally defined by the World Health Organization International Agency for Research on Cancer ${ }^{1}$ amended and updated based on current knowledge. The classification reflects relationships among lineage, prognosis (malignant, intermediate or locally aggressive, intermediate or rarely metastasizing), driver alterations, and additional parameters. Branch lengths determined by nearest neighbor joining of a discretized distance matrix based on aforementioned variables. Initial branching reflects differences in lineage with associated lineages appearing closer in distance (e.g. skeletal and smooth muscle). Subsequent branching encodes similarity in prognosis, whether they are translocation-associated, and if so, the genes shared among 
distinct fusions (in this order). While incomplete, as many subtypes lack sufficient global molecular profiling data on which to base a phylogeny, this initial formulation minimally reflects the relationships among lineage and major molecular lesions in the subtypes. The figure excludes 52 benign types of tumor. MFH, as abbreviated, represents undifferentiated pleomorphic sarcoma. 
A.

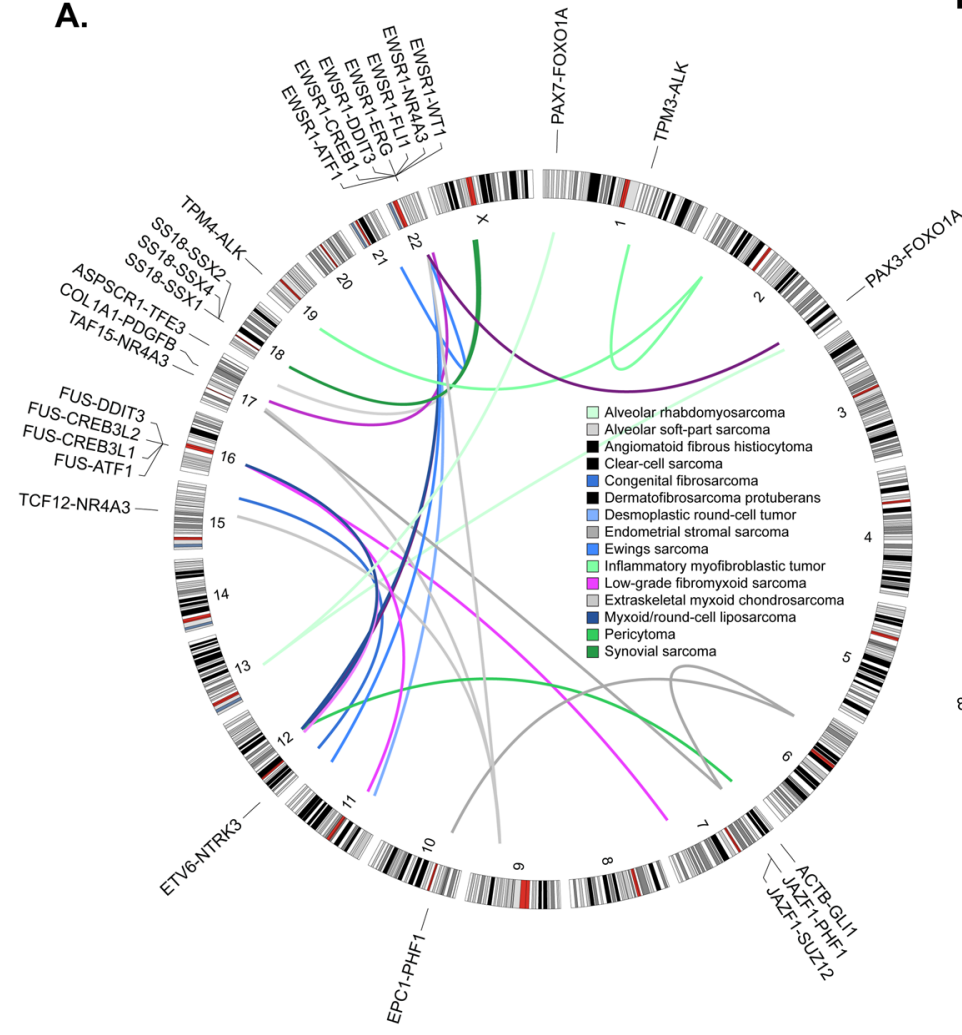

B.

Figure 2. The structure of sarcoma genomes

A, Summary of recurrent translocations in malignant soft-tissue sarcomas indicates shared fusion partners between subtypes and regions of the genome subject to more frequent rearrangement. The outer ring represents genomic location (as labeled), and curves join fusion partners. B, Upper right: the somatic structure of an intermediate-genomic complexity sarcoma, a dedifferentiated liposarcoma (whole genome, inset) as defined by long-insert low depth-of-coverage mate-paired second-generation sequencing (see Box 1; Taylor BS and Singer S, unpublished data). Intra-chromosomal rearrangements are shown in gold and interchromosomal rearrangements in red; a subset of the interchromosomal rearrangements is reminiscent of rearranged sequence on chromosome 12 (chr12) in panel A. Lower left: the pathognomonic chromosome $12 \mathrm{q}$ amplification is shown in greater detail. This detailed view indicates a dense network of back-and-forth inverted and non-inverted intra-chromosomal rearrangements in three clusters (in grey, light blue, and dark blue; for clarity, interchromosomal rearrangements excluded). The curves reflect rearrangements between two genomic loci as determined experimentally and computationally. ACTB, actin- $\beta ; A L K$, anaplastic lymphoma receptor tyrosine kinase; $A S P S C R 1$, alveolar soft part sarcoma chromosome region candidate 1; $A T F 1$, activating transcription factor 1; COL1A1, collagen type Ia 1; $C R E B 1$, cAMP responsive element binding protein 1; CREB3L, AMP responsive element binding protein 3-like; DDIT3, DNA-damage-inducible transcript 3 (also known as CHOP); EPC1, enhancer of polycomb homolog 1; ETV6, ets variant 6; EWSR1, Ewing sarcoma breakpoint region 1; FLI1, Friend leukemia virus integration 1; FOXO1, forkhead box O1; FUS, fused in sarcoma; GLI1, GLI family zinc finger 1; JAZF1, JAZF zinc finger 1; NR4A3, nuclear receptor subfamily 4A3; NTRK3, neurotrophic tyrosine kinase receptor 3; $P A X$, paired box; $P D G F B$, platelet-derived growth factor- $\beta ; P H F 1$, PHD finger protein 1 ; $S S 18$, synovial sarcoma translocation chromosome 18; SSX, synovial sarcoma, X breakpoint; $S U Z 12$, suppressor of zeste 12; TAF15, TAF15 RNA polymerase II TATA box 
binding protein-associated factor; TCF12, transcription factor 12; TFE3, transcription factor binding to IGHM enhancer 3; TPM, tropomyosin; WT1, Wilms tumor 1. 


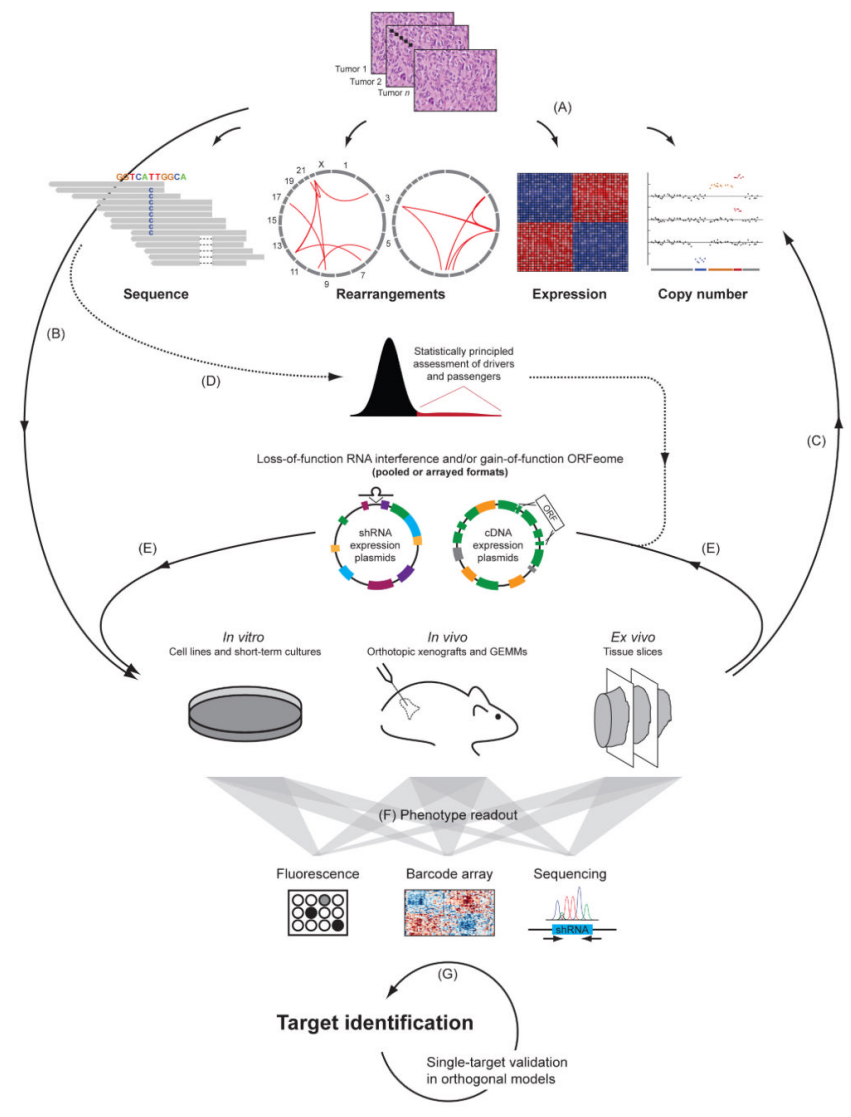

Figure 3. Models and functional genetics

High-throughput integrative genomics, increasingly dominated by second-generation sequencing, identifies abnormalities of sequence, structure, expression, or copy number (A) in sarcoma genomes (notwithstanding epigenetic modification). In parallel, model systems (cultured cells, animal models, or tissue slices) need to be generated from human tumors (B) and similarly genomically profiled to confirm that they represent primary tumors in the retention of driver alterations (C). From tumor profiles, computational methodologies analyze the patterns of recurrence to distinguish likely driver from passenger alterations (D) (Box 2). These models can then be subjected to high-throughput functional genetic analyses, including both gain-of-function approaches (such as open reading frame (ORF) overexpression with pLX-Blast-V5 or similar cDNA expression vectors) and loss-offunction approaches (such as RNA interference using pLKO1-puro or similar shRNA plasmids) (E). These approaches can be applied either to all genes (to identify genotypeselective targets from those that are not) or to those identified by the integrative genomic and statistical methods. The readouts of these high throughput methods, be it fluorescence, barcode arrays, or sequence read counts, provide data on one of a large number of possible phenotypes and can be analyzed (F) to identify genotype-dependent vulnerabilities in sarcoma cells, identifying targets that can be validated in orthogonal models $(\mathrm{G})$, a subset of which may be suitable for therapeutic intervention. This model of genomics-driven functional genetics focuses on rapid functional annotation of cancer genomes. GEMM, genetically engineered mouse model. 

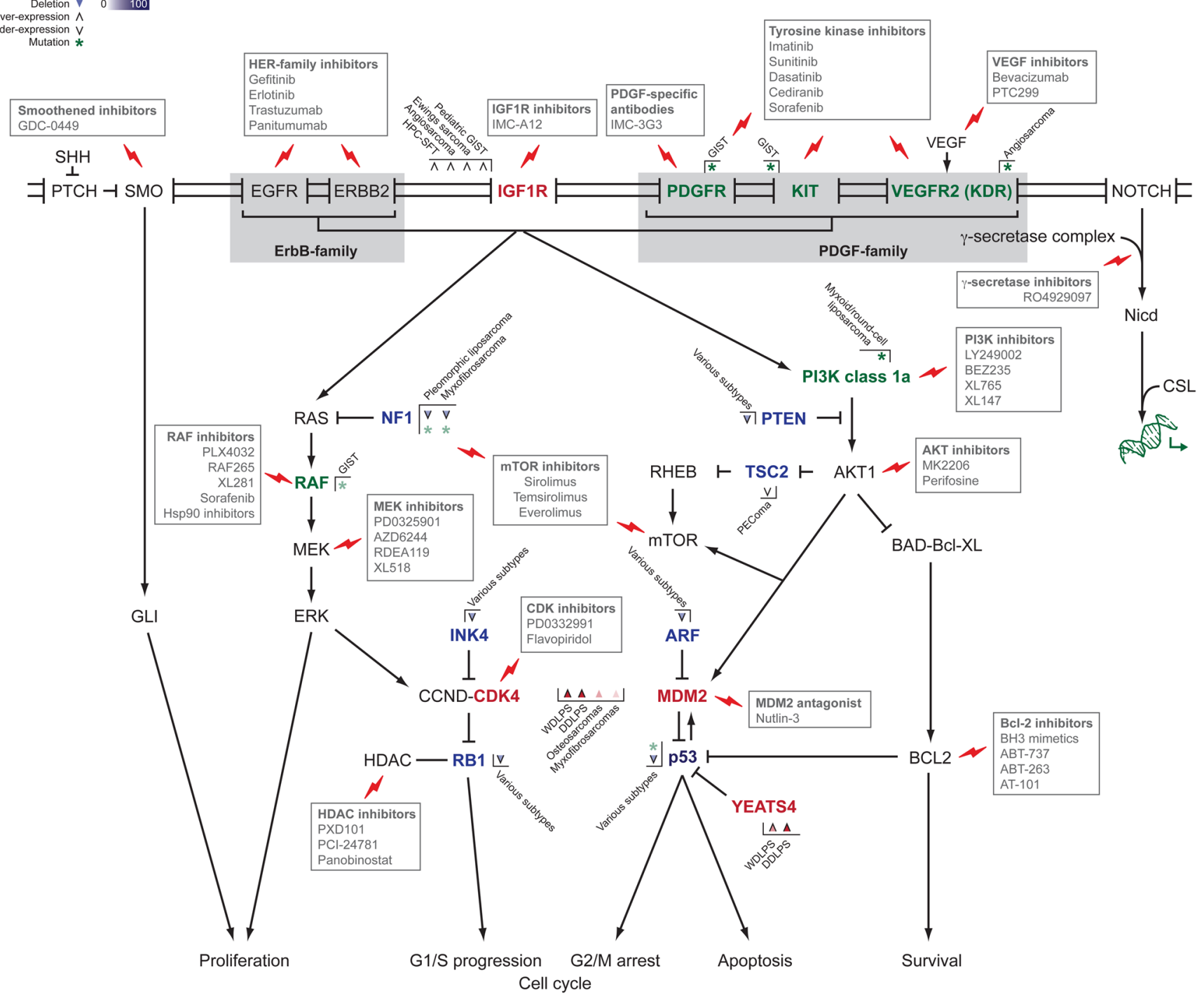

Figure 4. Pathways for targeted therapy in sarcoma

Diverse subtype-specific alterations imply that a variety of signaling pathways function aberrantly in sarcomas. Abbreviated pathways include Ras-Raf, PI3K, mTOR, p53, cell cycle and survival, Notch, and Hedgehog signaling, all of which are targeted by a growing list of specific therapies. Here, annotation of nodes in signaling networks affected by specific genomic abnormalities includes affected subtype, alteration types (genomic amplification or deletion are solid triangles, over- or under-expressed are open arrowheads, mutated are starred), and frequencies. A subset of nodes are colored by their dominant alteration type (see key). Targeted agents (gray) include those in clinical use and those in preclinical or early-phase development in sarcoma. CCND, cyclin D; CDK, cyclindependent kinase; CSL, recombination signal binding protein for immunoglobulin kappa $\mathbf{J}$ region (also known as RBPJ); DDLPS, dedifferentiated liposarcoma; EGFR, epidermal growth factor receptor; GIST, gastrointestinal stromal tumor; HDAC, histone deacetylase; HPC-SFT, hemangiopericytoma-solitary fibrous tumor; HSP90, heat shock protein 90; IGF1R, insulin-like growth factor 1 receptor; NICD, NOTCH intracellular domain; NF1, neurofibromin 1; PDGFR, platelet-derived growth factor receptor; PEComa, perivascular 
epitheliod cell tumor; PTCH, patched; RB1, retinoblastoma 1; RHEB, Ras homolog enriched in brain; SMO, smoothened; SSH, slingshot; TSC2, tuberin; VEGFR2, vascular endothelial growth factor receptor 2; WDLPS, well-differentiated liposarcoma. 

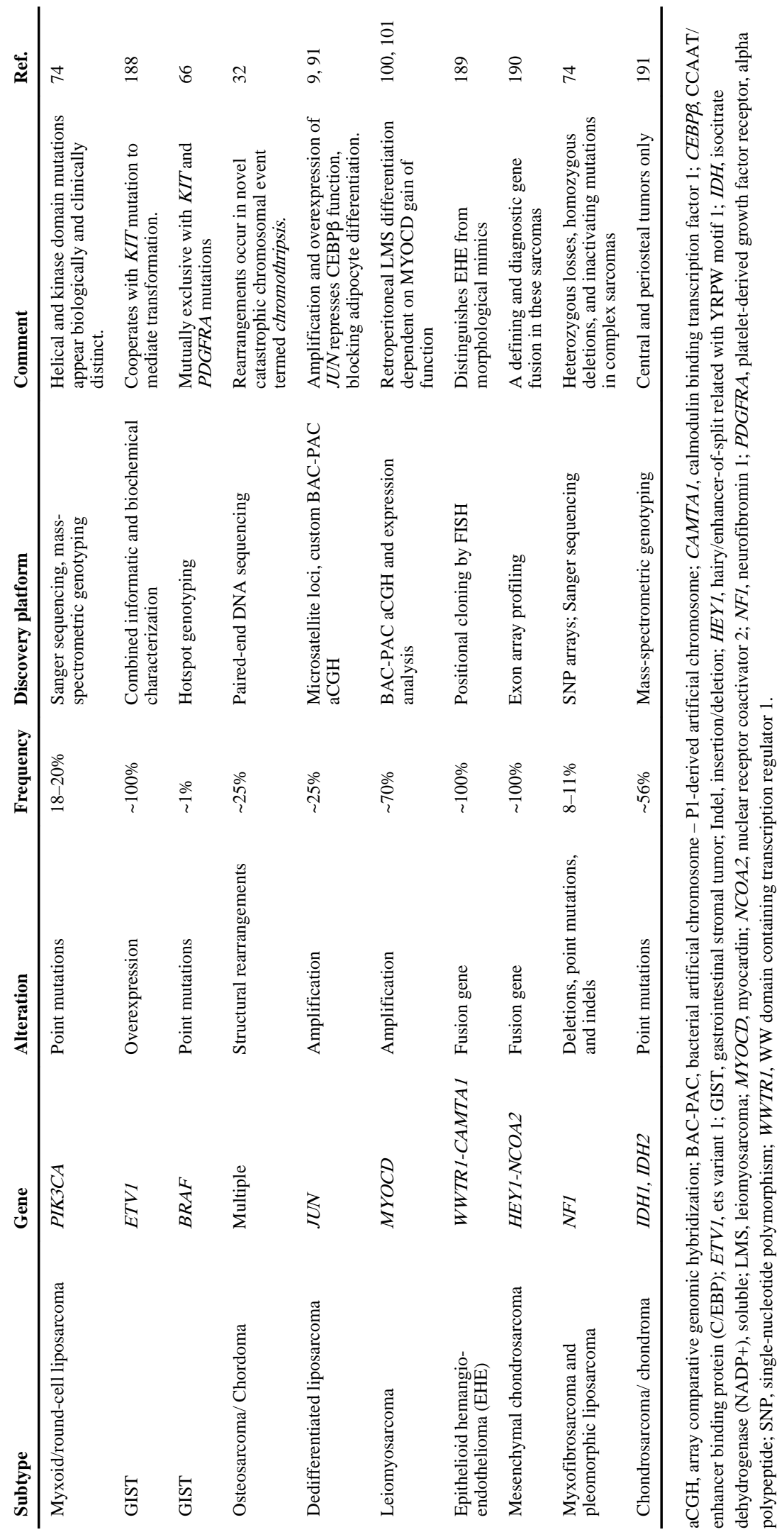

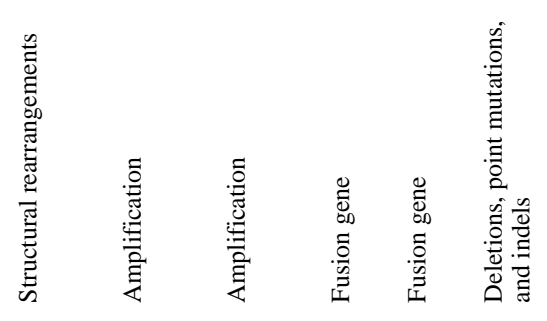
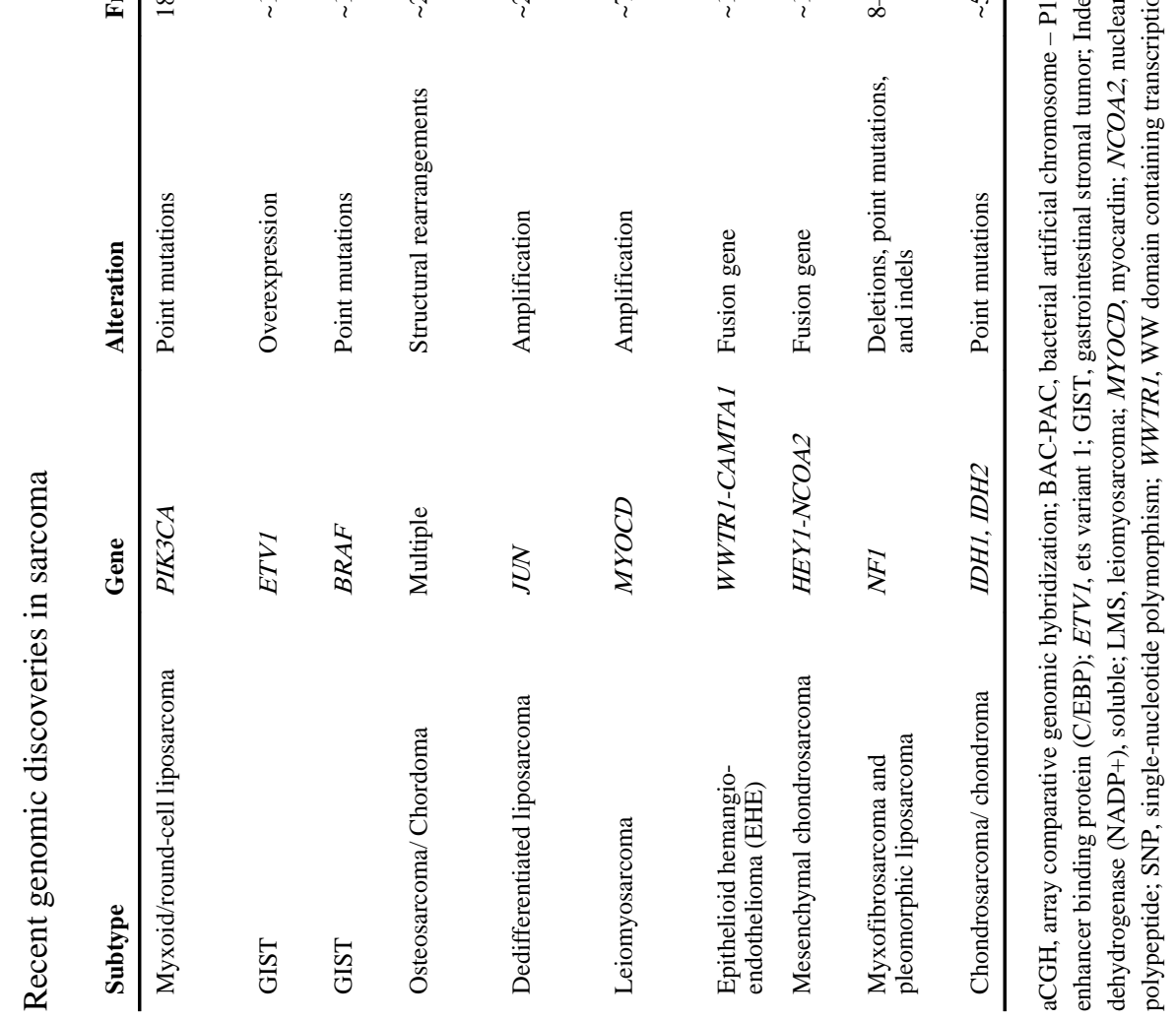


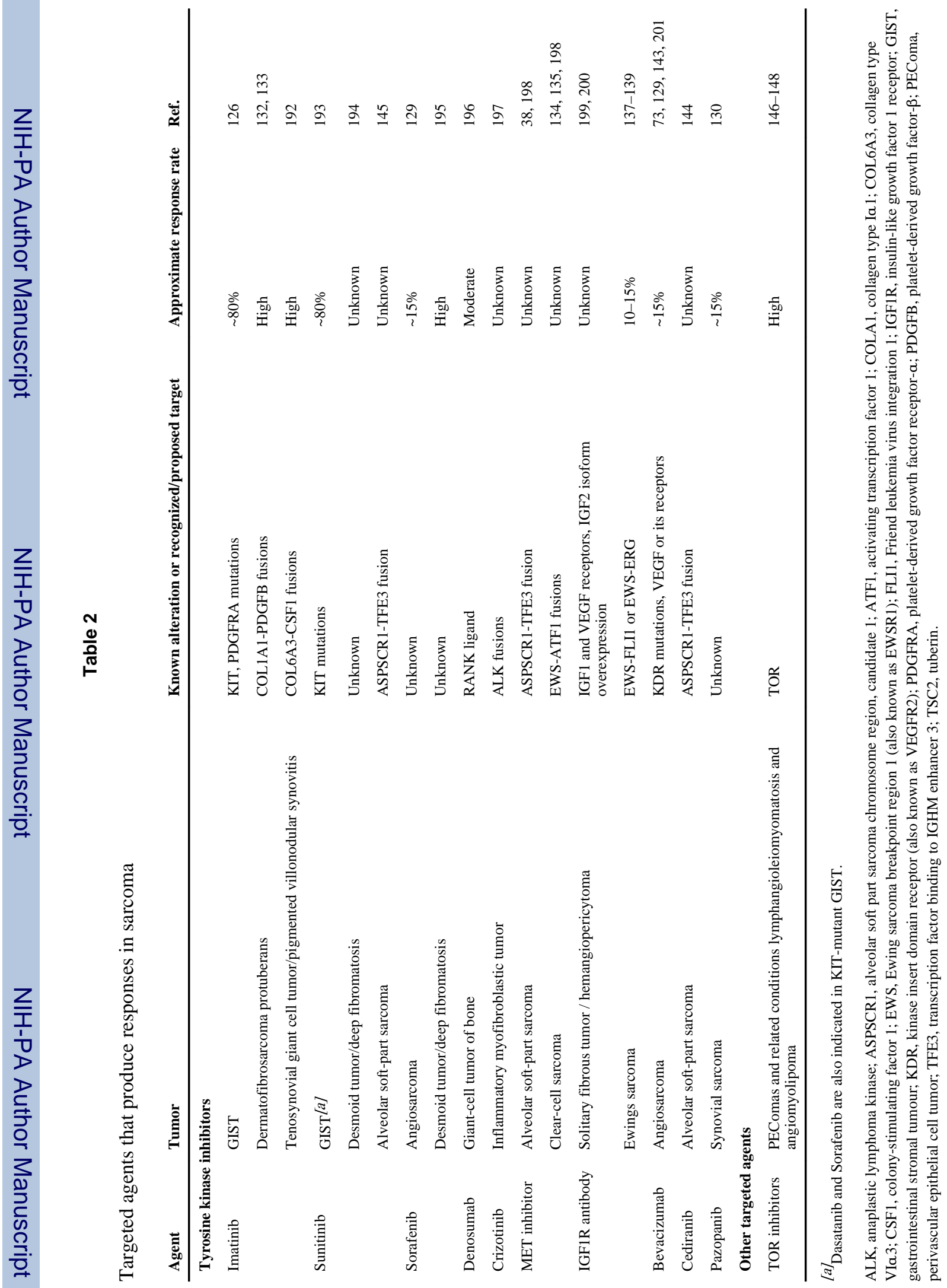

